\title{
Melanocortin-1 Receptor Positively Regulates Human Artery Endothelial Cell Migration
}

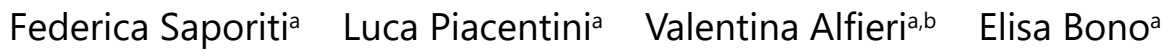 \\ Fabrizio Ferrari $^{\mathrm{a}}$ Mattia Chiesa $^{\mathrm{a}}$ Gualtiero I. Colombo $^{\mathrm{a}}$ \\ aUnit of Immunology and Functional Genomics, Centro Cardiologico Monzino IRCCS, Milan, Italy, \\ ${ }^{b}$ Department of Pharmacological and Biomolecular Sciences, Università degli Studi di Milano, Milan, \\ Italy
}

\author{
Key Words \\ Melanocortin receptors $\bullet \alpha-\mathrm{MSH} \cdot$ Human artery endothelial cells $\bullet$ Cell migration
}

\begin{abstract}
Background/Aims: Melanocortin receptors (MCRs) belong to a hormonal signalling pathway with multiple homeostatic and protective actions. Microvascular and umbilical vein endothelial cells (ECs) express components of the melanocortin system, including the type 1 receptor (MC1R), playing a role in modulating inflammation and vascular tone. Since ECs exhibit a remarkable heterogeneity, we investigated whether human artery ECs express any functional MCR and whether its activation affects cell migration. Methods: We used reverse transcription real-time PCR to examine the expression of melanocortin system components in primary human artery ECs. We assessed MC1R protein expression and activity by western blot, immunohistochemistry, CAMP production, and intracellular $\mathrm{Ca}^{2+}$ mobilization assays. We performed gap closure and scratch tests to examine cell migration after stimulation with alpha-melanocyte-stimulating hormone $(\alpha-M S H)$, the receptor highest-affinity natural ligand. We assessed differential time-dependent transcriptional changes in migrating cells by microarray analysis. Results: We showed that human aortic ECs (HAoECs) express a functionally active MC1R. Unlike microvascular ECs, arterial cells did not express the $\alpha-M S H$ precursor proopiomelanocortin, nor produced the hormone. MC1R engagement with a single pulse of $\alpha-\mathrm{MSH}$ accelerated HAoEC migration both in the directional migration assay and in the scratch wound healing test. This was associated with an enhancement in $\mathrm{Ca}^{2+}$ signalling and inhibition of CAMP elevation. Time-course genome-wide expression analysis in HAoECs undergoing directional migration allowed identifying dynamic co-regulation of genes involved in extracellular matrix-receptor interaction, vesicle-mediated trafficking, and metal sensing - which have all well-established influences on EC motility -, without affecting the balance between pro- and anticoagulant genes. Conclusion: Our work broadens the knowledge on peripherally expressed MC1R. These results indicate that the receptor is

F. Saporiti, L. Piacentini and V.Alfieri contributed equally to this work.

\begin{tabular}{ll}
\hline Gualtiero I. Colombo, M.D., & Head, Lab. of Immunology and Functional Genomics, Centro Cardiologico Monzino IRCCS \\
Ph.D. & Via Carlo Parea, 4-20138 Milano (Italy) \\
& Tel. +39 025800.2464 , Fax +39025800.2750, E-Mail gualtiero.colombo@cardiologicomonzino.it
\end{tabular}
\end{abstract}


constitutively expressed by arterial ECs and provide evidence of a novel homeostatic function for MC1R, whose activation may participate in preventing/healing endothelial dysfunction or denudation in macrovascular arteries.

\section{Introduction}

The melanocortin receptors (MCRs) are a family of rhodopsin-like G protein-coupled receptors (GPCRs) that are activated by different melanocortin peptide ligands, derived from the tissue-specific cleavage of a common preprohormone precursor, the proopiomelanocortin (POMC) [1]. These molecules, together with a number of endogenous antagonists and accessory proteins, constitutes the so-called melanocortin system [2]. To date, five MCRs have been identified, with different tissue distribution and a diverse affinity for their natural ligands. MCRs mainly signal through intracellular cAMP increase or, alternatively, transient intracellular elevation of cytosolic free $\mathrm{Ca}^{2+}$ [3]. The melanocortin system has been studied for its ability to regulate several physiological processes, including pigmentation, adrenocortical steroidogenesis, energy homeostasis, and exocrine gland secretion. In particular, the prototypical melanocortin peptide, the alpha-melanocyte stimulating hormone $(\alpha-\mathrm{MSH})$, possesses a wide spectrum of anti-inflammatory [4], immunoregulatory [5], and cytoprotective activities, including protection and repair after organ damage (i.e. cerebral and myocardial ischemia/reperfusion injury, nephrotoxicity, and acute lung injury) [6]. As a consequence, targeting melanocortin system is considered a promising strategy for new therapeutic approaches in various inflammatory conditions [7].

The melanocortin system has been involved in the modulation of oxidative stress [8] and vascular endothelial damage [9]. A local melanocortin system has been described in endothelial cells (ECs) of the cutaneous microcirculation [10]. Moreover, the MC1R (and no other MCR) has been detected both on murine brain microvascular ECs [11], and on human dermal microvascular ECs (HDMECs) $[10,12]$ and umbilical vein ECs (HUVECs) [13], with possible modulatory effects on endothelium homeostasis. In particular, $\alpha$-MSH has been shown to modulate blood vessel tone by enhancing nitric oxide-cyclic guanosine monophosphate dependent relaxation responses through endothelial MC1R [13]. Nonetheless, a formal demonstration that human artery ECs of the macrovasculature express functional MCR(s) is currently missing. This is substantial because ECs exhibit a remarkable heterogeneity and show specific structure and functions associated with the blood vessel they belong to, i.e. large and medium arteries, veins, or capillaries $[14,15]$. At the molecular level, ECs display phenotype markers that are cell type-restricted, and exhaustive genomewide expression studies have shown unique gene expression patterns in ECs derived from different tissues $[16,17]$. This heterogeneity accounts for many human vascular diseases restricted to specific types of vessels. Nevertheless, our knowledge of EC biology has been mostly inferred by studies on HUVECs, which are cells that originate from a vessel type that is rarely affected by vascular disorders [18]. HDMECs and HUVECs do not recapitulate the physiology of all the vascular ECs and, most importantly, their ability to activate specific cell functions in response to MCR ligands may not overlap those of artery ECs.

A recent report showed that treatment with MCR agonists was able to prevent the development of vascular dysfunction and attenuate plaque inflammation in a mouse model of pre-established atherosclerosis [19]. Artery endothelial dysfunction and/or injury are prominently linked to the pathogenesis of atherosclerosis, thrombosis, or surgery procedure complications [20]. An essential biological process involved in endothelial healing upon vascular injury is EC migration. When a blood vessel is damaged, the restoration of endothelium and vessel integrity is achieved through migration of healthy ECs to the site of the lesion and subsequent proliferation. Hence, EC migration has a key role, besides angiogenesis, in vascular repair and tissue regeneration [21]. In this work, we investigated whether human artery ECs express any functional MCR and whether MCRs activation through $\alpha$-MSH can affect artery EC migration. 


\section{Cellular Physiology Cell Physiol Biochem 2019;52:1339-1360

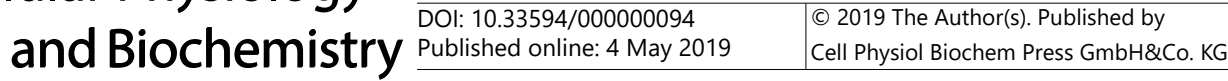 \\ Saporiti et al.: MC1R Enhances Endothelial Cell Migration}

\section{Materials and Methods}

\section{Primary human artery endothelial cells}

We purchased primary human artery ECs from the European Collection of Authenticated Cell Cultures (ECACC, Salisbury, UK), Lonza (Allendale, NJ), and Promocell (Heidelberg, Germany). We obtained three adult human aortic ECs (HAoECs) and recoded them as $c 1, c 2$ and $c 3$, namely: (c1) HAoEC (304-05a) from ECACC (catalogue no. 06090729), (c2) HAoEC from Lonza (catalogue no. CC-2535), and (c3) HAoEC from Promocell (catalogue no. C-12271). We also obtained three adult human coronary artery ECs (HCAECs) and recoded them as $c 4, c 5$ and $c 6$, namely: (c4) HCAEC (300-05a) from ECACC (catalogue no. 06090727), (c5) HCAEC from Lonza (catalogue no. CC-2585), and (c6) HCAEC from Promocell (catalogue no. C-12221). Primary ECs were tested for cell-type specific markers by the manufactures. Cells were positive for Factor VIII-related antigen or von Willebrand factor and CD31 expression, positive for acetylated low-density lipoprotein uptake, and negative for $\alpha$-actin expression. Cells were seeded in $75 \mathrm{~mL}$ plastic flasks (Corning, Tewksbury, MA) at a density of $2.5 \times 10^{3}$ cells $/ \mathrm{cm}^{2}$ and cultured following manufactures' instructions. We performed all experiments at cell passages 4-8. We tested cell cultures for mycoplasma contamination before any experiments, using the PCR-based Mycoplasma detection kit Venor GeM OneStep (Minerva Biolabs, Berlin, Germany).

\section{Chemicals}

The $\alpha$-MSH peptide was obtained from Phoenix Pharmaceuticals (Burlingame, CA); the peptide 153N-6 (H-[Met5,Pro6,D-Phe7,D-Trp9,Phe10]-MSH(5-13)) from Bachem (Bubendorf, Switzerland); isobutyl methylxanthine (IBMX), forskolin, PD0332991 isethionate, and thapsigargin from Sigma-Aldrich (St. Louis, MO); 1, 2-Bis(2-aminophenoxy)ethane-N,N,N',N'-tetraacetic acid tetrakis(acetoxymethyl ester) (BAPTA-AM) and 1-[6-[[(17ß)-3-Methoxyestra-1, 3,5(10)-trien-17-yl]amino]hexyl]-1H-pyrrole-2, 5-dione (U-73122) from Tocris Bioscience (Bristol, UK). The peptides $\alpha$-MSH and 153N-6 were dissolved in water. IBMX, forskolin, PD0332991, thapsigargin, BAPTA-AM, and U-73122 were dissolved in dimethyl sulfoxide (DMSO).

\section{Reverse transcription quantitative PCR (RT-qPCR) for melanocortin system components}

Total RNA was extracted from ECs grown to confluence, adding TRIzol Reagent (Invitrogen, Carlsbad, $\mathrm{CA}$ ) directly to the culture dishes. Given that some MCRs are single-exon intronless genes (i.e., MC3R and MC4R), while others are multi-exon genes with several splice variants (e.g., MC1R [22]), we treated RNA samples with RNase-free DNase-I to eliminate genomic contamination and prevent amplification of genomic DNA. This allowed us to use a single-exon probe qPCR design to detect the canonical primary transcripts of the MCR genes. RNA quantification and purity assessment were performed by microvolume spectrophotometry on an Infinite M200 PRO multimode microplate reader (Tecan, Männedorf, Switzerland). RNA quality and integrity were checked by microfluidics electrophoresis with the RNA 6000 Nano Assay Kit on a 2100 Bioanalyzer (Agilent Technologies, Santa Clara, CA). Complementary DNA (cDNA) for single target gene expression analysis was synthesized from $2 \mu \mathrm{g}$ of total RNA for each sample using the High Capacity cDNA Reverse Transcription Kit (Applied Biosystems, Foster City, CA). TaqMan Array Human Endogenous Controls 96-Well Plate PCR assay (Applied Biosystems) was preliminarily employed to identify the most appropriate endogenous control gene. Analysis of gene expression stability and selection of the best reference gene was performed using the NormFinder v0.953 Excel Add-In [23]. We used single tube TaqMan Gene Expression Assays (Applied Biosystems) for evaluating mRNA expression of the melanocortin receptors (MCRs), proopiomelanocortin (POMC), prohormone convertases, and the endogenous constitutive gene (see details in Supplemental Methods - for all supplemental material see www.cellphysiolbiochem. com). Assays IDs for the melanocortin system components and the reference gene were Hs00267168_s1 $(M C 1 R), H s 00265039 \_s 1(M C 2 R), H s 00252036 \_s 1(M C 3 R), H s 00271877$ s1 (MC4R), Hs00271882_s1 (MC5R), Hs01596743_m1 (POMC), Hs01026107_m1 (proprotein convertase subtilisin/kexin type 1, PCSK1), Hs01037347_m1 (PCSK2), Hs00159829_m1 (furin, PCSK3), Hs00159844_m1 (PCSK6), Hs00161638_m1 (secretogranin V, SCG5), and Hs99999902_m1 (ribosomal protein large P0, RPLP0). We run three replicates of each assay for each sample (20 ng/well of cDNA) on a ViiA 7 Real-time PCR System (Applied Biosystems). Experimental threshold and baseline were imputed by algorithms implemented in the ViiA 7 software v1.2 (Applied Biosystems), and data were analysed by the Pfaffl's corrected $\Delta \Delta$ Ct method [24]. 


\section{Cellular Physiology Cell Physiol Biochem 2019;52:1339-1360

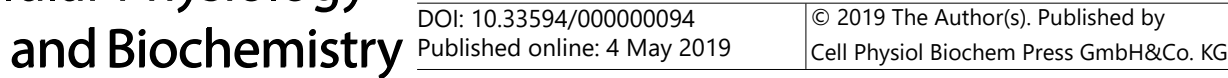 \\ Saporiti et al.: MC1R Enhances Endothelial Cell Migration}

$\alpha-M S H$ assay

Quantification of $\alpha$-MSH release by primary HAoECs and HCAECs was performed using an ultrasensitive fluorescent enzyme immunoassay (EIA) kit (Phoenix Pharmaceuticals), following manufacture's instruction. The EIA sensitivity, i.e. the minimum detectable concentration, was $8.9 \mathrm{pg} / \mathrm{mL}$. Cross-reactivity with the adrenocorticotropic hormone (ACTH) was zero: $\alpha$-MSH shares the sequence of ACTH (1-13), but $\alpha$-MSH is acetylated at the $\mathrm{N}$-terminus and amidated at the $\mathrm{C}$-terminus [7]. Cells were seeded to confluence in 96-well culture plates, in complete endothelial growth medium (EGM2; Lonza), and supernatants were collected and stored at $-80{ }^{\circ} \mathrm{C}$ until measurement.

\section{Genomic DNA sequencing for MC1R}

We used a 3500 Genetic Analyzer (Applied Biosystems) to perform DNA sequencing of the MC1R gene open reading frame (ORF) for all HCAECs and HAoECs. Genomic DNA amplicons of the MC1R ORF were produced by PCR with the following primers: MC1R_Forward(1) (-25) 5'-TCCTTCCTGCTTCCTGGACA-3', MC1R_Reverse(1) (+980) 5'-CACACTTAAAGCCGCGTGCAC-3'. The amplified fragments were purified using the Agencourt AMPure XP kit (Beckman Coulter). Sequencing reactions were carried out using the BigDye Terminator v3.1 Kit (Applied Biosystems) in both strand directions to allow the production of four overlapping fragments. Sequencing primers used were the MC1R_Forward(1), the MC1R_Reverse(1) and: the inner MC1R_Forward(2) (+449) 5'-TGCGCTACCACAGCATCGTG-3', and the inner MC1R_Reverse(2) $(+510)$ 5'-CACCCAGATGGCCGCAAC-3'. Unincorporated fluorescent dideoxynucleotides and salts were removed with the BigDye XTerminator Purification Kit (Applied Biosystems). The purified sequencing reaction products were electrokinetically injected into a $50 \mathrm{~cm}$ Capillary Array filled with the POP-7 Polymer (Applied Biosystems). Electropherograms were analysed by the Variant Reporter software v1.1 (Applied Biosystems).

\section{Antibodies}

Primary antibodies used were: anti-MC1R rabbit polyclonal antibody, supplied with the specific control peptide antigen (Alomone Labs, Jerusalem, Israel); anti-ATPase $\mathrm{Na}+\mathrm{K}+$ transporting subunit alpha 1 (ATP1A1) rabbit polyclonal antibody (Cell Signaling Technology, Danvers, MA); anti- $\beta$-actin mouse monoclonal IgG1 (Novus Biologicals, Littleton, C0); and anti-Ki67 rabbit polyclonal IgG (Abcam, Cambridge, UK). Secondary antibodies were: donkey anti-rabbit or anti-mouse IgG conjugated, respectively, to IRDye 800CW and IRDye 680RD infrared dyes (LI-COR Biosciences, Lincoln, NE), for immunoblotting; donkey anti-rabbit IgG conjugated to the DyLight 488 fluorochrome (Jackson ImmunoResearch Laboratories, West Grove, PA), for immunocytochemistry.

\section{Western blotting}

HAoECs and HCAECs $\left(1.2 \times 10^{6}\right)$ were lysed in Milliplex MAP Lysis buffer (Millipore, Billerica, MA) with a complete protease inhibitor cocktail (Roche, Mannheim, Germany) to obtain whole extracts, or with the FractionPREP Cell Fractionation Kit (BioVision, Milpitas, CA) to obtain plasma membrane extracts. Proteins were quantified using the Pierce BCA Protein Assay Kit (Thermo Fisher Scientific). Thirty $\mu \mathrm{g}$ of each protein extract were mixed with the Novex Tris-Glycine SDS sample buffer $2 \times$ and the Novex sample reducing agent 10× (Invitrogen). Samples were loaded onto 4-12\% gradient Novex WedgeWell precast TrisGlycine polyacrylamide gels (Invitrogen) and run in Novex Tris-Glycine SDS running buffer for 40 min at 200 V. Samples were blotted on nitrocellulose membranes using an iBlot system (Invitrogen). Membranes were blocked in the Odyssey blocking buffer (LI-COR Biosciences) for $1 \mathrm{~h}$. Pre-absorption was performed by incubating the anti-MC1R antibody for $30 \mathrm{~min}$ at room temperature with the inhibitory MC1R peptide (two-fold excess of the peptide by weight). Primary or the pre-absorbed antibodies were diluted (1:1000) in the Odyssey blocking buffer (LI-COR Biosciences), and membranes were incubated overnight at $4^{\circ} \mathrm{C}$. Anti- $\beta$-actin and anti-ATP1A1 antibodies (1:5000) were used as reference controls for whole or membrane extracts, respectively. Membranes were incubated with IRDye secondary antibodies (1:10000) for 20 min at room temperature. Immunoreactive bands were detected by an Odyssey Infrared Imaging System (LI-COR Biosciences). 


\section{Cellular Physiology Cell Physiol Biochem 2019;52:1339-1360 \begin{tabular}{ll|l} 
and Biochemistry & Dublished online: 4 May 2019 & $\begin{array}{l}\text { P } 2019 \text { The Author(s). Published by } \\
\text { Cell Physiol Biochem Press GmbH\&Co. KG }\end{array}$ \\
\hline
\end{tabular} \\ Saporiti et al.: MC1R Enhances Endothelial Cell Migration}

Anti-MC1R antibody specificity testing

The anti-MC1R antibody was raised against an epitope corresponding to amino acid residues 217-232 in the $3^{\text {rd }}$ intracellular loop of human MC1R. To test the specific binding of the anti-MC1R antibody to the MC1R protein, we generated a positive control for subsequent analyses by transiently transfecting human HEK293 cells with the MC1R full-length cDNA by a C-terminal fusion of tGFP tag in a pCMV6 vector (Origene, Rockville, MD). Cells were grown in RPMI 1640 with $10 \%$ foetal bovine serum (FBS), penicillin $100 \mathrm{U} / \mathrm{mL}$ and streptomycin $10 \mu \mathrm{g} / \mathrm{mL}$ (Sigma-Aldrich) to approximately 50\% confluence, and then transfected by incubation with the TransIT-LT1 Transfection Reagent (Mirus Bio, Madison, WI) for $48 \mathrm{~h}$. Specificity of the MC1R antibody was demonstrated by pre-absorption with the specific blocking peptide supplied with the primary antibody, which abolished MC1R signal in Western immunoblot (see Supplementary Fig. S1).

\section{Intracellular cAMP assay}

Quantification of intracellular cAMP levels was performed using the cAMP Biotrak enzyme immunoassay (GE Healthcare Life Sciences, Piscataway, NJ). HAoECs were seeded to confluence in 96-well plates or 24-well plates with IBIDI culture inserts (Martinsried, Germany). Cells, prior to 5-min stimulation with $\alpha$-MSH $10^{-8} \mathrm{M}$, were pre-treated for $30 \mathrm{~min}$ with IBMX $0.1 \mathrm{mM}$, to inhibit cAMP degradation by phosphodiesterases (PDEs). Cells treated with IBMX alone were used as negative controls, whereas cells stimulated with the activator of eukaryotic adenylyl cyclase forskolin $(10 \mu \mathrm{M})$ served as positive controls. As control for receptor-binding specificity, cells were pre-treated with the MC1R-selective competitive $\alpha$-MSH antagonist $153 \mathrm{~N}-6\left(10^{-5} \mathrm{M}\right)[25,26]$ for $15 \mathrm{~min}$ in separate experiments. The abovementioned concentration of $\alpha$-MSH was selected for all functional assays based on previous publications on HDMECs $[10,12]$ and on pilot experiments with 100 -fold scalar concentrations of peptide $\left(10^{-6} \mathrm{M}, 10^{-8} \mathrm{M}\right.$, and $\left.10^{-10} \mathrm{M}\right)$ that showed its effectiveness (see Results below).

\section{Immunohistochemistry for MC1R}

Formaldehyde-fixed paraffin sections of a normal human aorta were incubated with the primary antiMC1R antibody overnight at $4^{\circ} \mathrm{C}$. As control of the staining specificity, the anti-MC1R antibody was preincubated $30 \mathrm{~min}$ with its specific blocking peptide. Slides were incubated with a biotinylated goat antirabbit IgG secondary antibody (1:200; Vector Laboratories, Burlingame, CA) and signals were revealed using the VECTASTAIN Elite ABC-HRP kit combined with the ImmPACT DAB EqV peroxidase (HRP) substrate (Vector Laboratories). Images were recorded using an AxioSkop microscope equipped with an AxioCam camera (Carl Zeiss).

\section{Directional cell migration assay}

The live-cell staining, lipophilic, near-infrared fluorescent membrane probe 1, 1'-dioctadecyl-3, $3,3^{\prime}, 3^{\prime}$-tetramethylindotricarbocyanine iodide (DiR) was used for imaging of gap closure in a cell migration assay [27]. HAoECs were treated with a solution of $2.5 \mu \mathrm{M}$ DiR (Biotium, Hayward, CA) in complete EGM2 medium for $20 \mathrm{~min}$ at $37^{\circ} \mathrm{C}$, washed and seeded onto 24-well plates with culture inserts (IBIDI, Martinsried, Germany). Inserts were removed to create a cell-free gap of approximately $500 \mu \mathrm{m}$, and HAoECs were allowed to migrate for $12 \mathrm{~h}$ at $37^{\circ} \mathrm{C}$ and $5 \% \mathrm{CO}_{2}$ in the presence of $10^{-8} \mathrm{M} \alpha$ - $\mathrm{MSH}$ or in medium alone. As control for receptor-binding specificity, cells were pre-treated with the MC1R-selective antagonist $153 \mathrm{~N}-6\left(10^{-5} \mathrm{M}\right)$ for $15 \mathrm{~min}$. In addition, to dissect the calcium-dependency of the $\alpha$-MSH-induced cell migration, experiments were repeated pre-treating cells for 15 min with either the intracellular $\mathrm{Ca}^{2+}$ chelator BAPTA-AM $\left(10^{-5} \mathrm{M}\right)$ or the phospholipase C (PLC) inhibitor U-73122 $\left(5 \times 10^{-5} \mathrm{M}\right)$. Plates were scanned with the Odyssey imaging system (LI-COR Biosciences) at $0,3,9$, and $12 \mathrm{~h}$, at $84 \mu \mathrm{m}$ resolution and high quality setting (emission, 800 $\mathrm{nm})$. Scans were converted to 8-bit images and analysed with the NIH ImageJ software v1.38x. For timecourse gene-expression analysis, $2 \times 10^{4}$ HAoECs were plated in high $35-\mathrm{mm}$ dishes with culture inserts (IBIDI) and treated with $\alpha-\mathrm{MSH} 10^{-8} \mathrm{M}$.

\section{Scratch wound healing assay}

HAoEC migration was also assessed using a scratch migration assay. Briefly, HAoECs were seeded onto 6-well tissue culture plates at a density of $2.5 \times 10^{3} \mathrm{cells} / \mathrm{cm}^{2}$ and grown to confluence. A gap of approximately $1 \mathrm{~mm}$ was created in the adherent layer of confluent ECs by using a sterile 0.1 -mL pipette tip. 


\section{Cellular Physiology Cell Physiol Biochem 2019;52:1339-1360

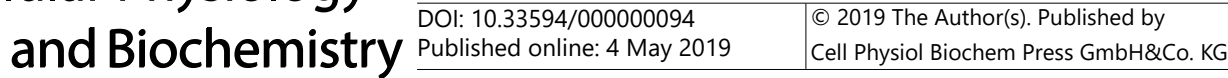 \\ Saporiti et al.: MC1R Enhances Endothelial Cell Migration}

After treatment with medium alone or $\alpha$-MSH $10^{-8} \mathrm{M}$, with or without 15-min pre-treatment with the MC1R antagonist $153 \mathrm{~N}-6\left(10^{-5} \mathrm{M}\right)$, the closure extent of the cell-free gap was detected by confocal microscope imaging (Zeiss, Jena, Germany) at 6 and $24 \mathrm{~h}$ and measured using the NIH ImageJ software v1.38x.

\section{Immunostaining for Ki67}

HAoECs were plated in 8-chamber $\mu$-slides (IBIDI) at a density of $1.0 \times 10^{3}$ cells $/ \mathrm{cm}^{2}$, treated with medium alone, $\alpha$-MSH $10^{-8} \mathrm{M}$, or $\alpha$-MSH plus $153 \mathrm{~N}-6$, and allowed to migrate for $24 \mathrm{~h}$. Cells were then fixed for $20 \mathrm{~min}$ in 4\% paraformaldehyde solution in PBS and permeabilised with 0.1\% Triton X-100 (Sigma-Aldrich). Non-specific antibody binding was prevented by using a blocking solution of $10 \%$ normal donkey serum (Jackson ImmunoResearch Laboratories) for $1 \mathrm{~h}$. Cells were incubated with the anti-Ki-67 primary antibody (1:100) overnight at $4^{\circ} \mathrm{C}$ and, then, with the DyLight-conjugated species-specific secondary antibody (1:500) for $2 \mathrm{~h}$ at room temperature. Slides were finally incubated with DAPI (Sigma-Aldrich; 1:1000) for 5 min to stain cell nuclei, mounted in a fluorescence mounting medium (Dako, Glostrup, Denmark), and examined with an ApoTome fluorescence microscope (Carl Zeiss, Jena, Germany). Images were acquired using the ZEN software v.5.0 SP1.1 (Carl Zeiss) and analysed with the ImageJ software, counting the percentage of Ki-67 positive cells over the total number of nuclei in 10 different fields for each treatment conditions in 4 independent experiments.

\section{Cell morphology assessment}

HAoECs were plated in 8-chamber $\mu$-slides (IBIDI) at a density of $1.0 \times 10^{3}$ cells $/ \mathrm{cm}^{2}$, incubated with $\alpha$-MSH $10^{-8} \mathrm{M}$ or medium alone for $6 \mathrm{~h}$, fixed for $10 \mathrm{~min}$ in $4 \%$ paraformaldehyde solution, and permeabilised with $0.1 \%$ Triton X-100 for $1 \mathrm{~h}$. Non-specific binding was prevented using a blocking solution of $5 \%$ bovine serum albumin. Cells were stained for $1 \mathrm{~h}$ at room temperature with phalloidin, a high-affinity probe for polymeric F-actin, conjugated to the red-orange fluorescent dye tetramethylrhodamine B isothiocyanate (TRITC) (Sigma-Aldrich). Slides were then stained with DAPI and images were acquired with an ApoTome fluorescence microscope (Carl Zeiss). Images were then analysed using the ZEN software and cell shape and stress fibres alignment were assessed. Changes in cell morphology were assessed by the ImageJ software measuring the major and minor cellular axis. Cells with axial ratios (long axis/short axis) larger than 3 were counted in randomly selected fields in 3 separate experiments and expressed as percentages of the total cells counted ( 250 cells on average).

\section{Intracellular $\mathrm{Ca}^{2+}$ mobilization assay}

Intracellular $\mathrm{Ca}^{2+}$ levels were measured using the Fluo-4 NW Calcium Assay Kit (Invitrogen). HAoECs, seeded onto a 24-well plate with IBIDI culture inserts in a calcium free medium, were loaded with $400 \mu \mathrm{L}$ of Fluo-4 NW for $30 \mathrm{~min}$ at $37^{\circ} \mathrm{C}$ and $5 \% \mathrm{CO}_{2}$. Fluorescence was measured for $300 \mathrm{sec}$ after treatment with $\alpha$-MSH $10^{-8}$ M using the Infinite M200 PRO plate reader (excitation, $494 \mathrm{~nm}$; emission, $516 \mathrm{~nm}$ ). Thapsigargin (10-8 M), an inhibitor of sarco/endoplasmic reticulum $\mathrm{Ca}^{2+}$-ATPases that causes a rapid raise of cytosolic $\mathrm{Ca}^{2+}$ by depleting endoplasmic reticulum stores [28], was used as positive control for $\mathrm{Ca}^{2+}{ }_{\mathrm{i}}$ release, treating HAoECs for $120 \mathrm{sec}$ before stimulation with $\alpha$-MSH $\left(10^{-8} \mathrm{M}\right)$. MC1R specific activation was assessed pretreating cells with the MC1R-selective antagonist $153 \mathrm{~N}-6\left(10^{-5} \mathrm{M}\right)$ for $15 \mathrm{~min} . \mathrm{Ca}^{2+}{ }_{\mathrm{i}}$ changes were calculated as the difference between the area under the curve (AUC) before (resting levels) and after addition of stimuli.

\section{Time-course gene expression analysis by microarray}

To isolate RNA from cells undergoing directional migration assay, we used the Agencourt RNAdvance cell v2 kit (Beckman Coulter, Beverly, MA), following manufacturer's instructions. RNA extracted from migrating HAoECs at $0.5,3,6$, and $12 \mathrm{~h}$ was used for microarray analysis. Labelled, linearly amplified complementary RNA (cRNA) was generated by Illumina Total Prep RNA Amplification Kit (Life Technologies), according to manufacturer's manual. Briefly, 200 ng of total RNA was reverse-transcribed to cDNA using an oligo(dT) primer containing a T7 promoter sequence. Second-strand cDNA was subsequently synthesized, and then in vitro transcribed adding biotin-dNTPs. After column-based purification and ammonium acetate/ethanol precipitation, cRNA was quantified by the Infinite M200 PRO plate reader. cRNA profile of all samples was checked by the RNA 6000 Nano Assay kit in an Agilent 2100 Bioanalyzer. cRNA (750 ng per sample) was hybridized at $58^{\circ} \mathrm{C}$ for $18 \mathrm{~h}$ on HumanHT-12 v4 Expression BeadChips (Illumina, San Diego, CA), followed by detection signal reaction with the fluorolink streptavidin-Cy3 (GE Healthcare Life Sciences) as recommended 


\section{Cellular Physiology Cell Physiol Biochem 2019;52:1339-1360

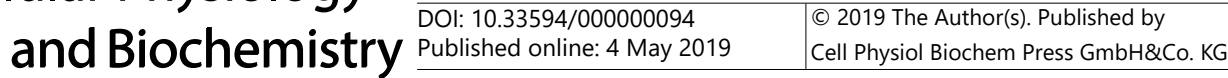 \\ Saporiti et al.: MC1R Enhances Endothelial Cell Migration}

by manufacturer's instructions. Each array on the BeadChips was scanned using an iSCAN System (Illumina). Array data export and quality control analysis were performed with the GenomeStudio Software v2011.1 (Illumina). Pre-processing of raw data was done by importing and analysing them with the lumi package [29], in the R software environment v2.15.2. Data variance stabilization was performed by variance stabilizing transformation (VST). Transformed data were normalized by robust spline normalization (RSN) algorithm, which combines the features of quantile and loess normalization. For subsequent analysis, we retained probes with a detection p-value $<0.01$ in at least $10 \%$ samples.

Raw and normalized, MIAME compliant microarray data are available in the NCBI's GEO repository under the accession number GSE49348 (http://www.ncbi.nlm.nih.gov/geo/query/acc.cgi?acc=GSE49348).

Microarray data were validated investigating mRNA expression of 84 wound healing related genes at different time points by RT-qPCR, using the Human Wound Healing RT ${ }^{2}$ Profiler PCR Arrays (Qiagen Sciences, Frederick, MD) following manufacturers' recommendations. The concordance of microarray hybridization intensities ( $\log _{2}$ transformed) with PCR data $(\mathrm{Ct})$ was measured computing the Pearson correlation coefficient and assessing its statistical significance.

\section{Statistical analysis and bioinformatics}

Data from functional assays passed the Shapiro-Wilk test for normality. Differences among groups were assessed by one-way ANOVA followed by Tukey's multiple comparisons test, or two-way ANOVA followed by Bonferroni post-hoc test, as appropriate. P-values $<0.05$ were considered statistically significant.

To analyse the time-course microarray gene-expression experiments, we used the Short Time-series Expression Miner (STEM) v1.3.8 algorithm [30] (see Supplementary Table S1 for analysis parameters). STEM implements a clustering method that lines up two steps. First, it selects a set of unique representative temporal profiles that, independently of the data, cover every possible expression profiles that can be generated in the experiment for a given set of parameters; second, it assigns to one of these temporal profiles only those gene profiles that pass the filtering criteria, as determined by a correlation coefficient. A permutation test was used to identify which profiles had a statistically significant enriched number of genes (for a false discovery rate, FDR $<0.05$ ), and significant profiles were grouped into larger clusters by their correlation degree $(\geq 0.7)$. As data input for STEM we used the $\log _{2}$ ratio of the $\alpha$-MSH-treated to nontreated HAoEC gene-expression values at each time point. Data were from three independent experiments, and each input derived from the average expression values of two technical hybridization replicates. The extent of the regulation is calculated as the maximum-to-minimum fold-change showed by the gene during the time-course.

Hierarchical clustering was performed using the GENE-E software v3.0.206 (https://software. broadinstitute.org/GENE-E). Unsupervised analysis of the average gene expression ratios of $\alpha$-MSH stimulated cells to non-stimulated cells, at the four time points considered, was performed using one minus Pearson correlation distance and the average linkage method.

Analysis of functional relations among regulated genes was made using the DAVID Bioinformatics Resources v6.7 (http://david.abcc.ncifcrf.gov/home.jsp) [31], testing for multiple annotations, i.e. Gene Ontology (GO) terms, KEGG pathways, and the Swiss Prot (SP)-Protein Information Resource (PIR) keywords. Redundant GO terms were removed using the web-based tool REViGO [32]. A network map of the enrichment analysis was produced by the Cytoscape program v2.8.2 [33], using the Enrichment Map app [34], a network-based visualization method for gene-set enrichment results.

\section{Results}

Human macrovascular endothelial cells constitutively express a functional MC1R, but not POMC

To determine which elements of the melanocortin system are expressed in human ECs from large vessels, we first performed real-time PCR analysis for detecting specific mRNAs in six human primary cells grown to confluence, i.e. three aortic ECs (HAoECs) and three coronary artery ECs (HCAECs). All the macrovascular ECs clearly expressed $M C 1 R$, but no other known MCRs (Table 1). Cell lines expressed the receptor mRNA at comparable levels, with the exception of one HCAEC which showed levels twice as high as the other HCAECs. 
Table 1. Expression of the MCRs, POMC, and prohormone convertases in primary human macrovascular endothelial cells. HAoEC: human aortic endothelial cells; HCAEC: human coronary artery endothelial cells; HA: human astrocytes (positive control). Three different primary lines for each type of EC were analysed by RT-qPCR: from ${ }^{c 1, c 4}$ ECACC, ${ }^{c 2, c 5}$ Lonza, and ${ }^{c 3, c 6}$ Promocell. In the upper panel, detection levels are reported as: -, undetected;,$+<35 \mathrm{Ct}$; and,$++<30 \mathrm{Ct}$. In the lower panel, MC1R expression levels in each cell line are shown as means $\pm \mathrm{SEM}$ of triplicate technical replicates

\begin{tabular}{lccccccccccc}
\hline Cell & MC1R & MC2R & MC3R & MC4R & MC5R & POMC & PCSK1 & PCSK2 & PCSK6 & FURIN & SCG5 \\
\hline HAoEC $\mathrm{c} 1, \mathrm{c} 2, \mathrm{c} 3$ & ++ & - & - & - & - & - & + & - & + & + & + \\
HCAEC $\mathrm{c} 4, \mathrm{c} 5, \mathrm{c} 6$ & ++ & - & - & - & - & - & + & - & + & + & + \\
HA & ++ & - & - & + & - & + & ++ & ++ & ++ & ++ & + \\
\hline & & HAoEC $\mathrm{c} 1$ & & HAoEC $\mathrm{c} 2$ & HAoEC $\mathrm{c} 3$ & HCAEC $\mathrm{c} 4$ & & HCAEC $\mathrm{c} 5$ & HCAEC $\mathrm{c} 6$ \\
\hline $\begin{array}{l}\text { MC1R normalized } \\
\text { expression levels }\end{array}$ & \multirow{2}{*}{$0.95 \pm 0.29$} & & \multirow{2}{*}{$1.16 \pm 0.11$} & $1.00 \pm 0.19$ & $0.71 \pm 0.09$ & & \multirow{2}{*}{$1.63 \pm 0.06$} & $0.81 \pm 0.04$ \\
\hline
\end{tabular}

Fig. 1. HAoECs express a functional MC1R. (A) Immunoblot analysis of membrane extracts showed that all three studied primary cells express MC1R on the plasma membrane. An anti-ATPase $\mathrm{Na}+\mathrm{K}+$ transporting subunit alpha 1 (ATP1A1) antibody was used as membrane-specific loading control (upper light grey arrow). Absence of a $\beta$-actin immunoreactive band (lower light grey arrow) excluded contamination with cytoplasmic proteins in this preparation. The $37 \mathrm{kDa}$ MC1Rspecific immunoreactive band is indicated by a grey arrow. Lanes are: $\mathrm{M}$, molecular weight marker; C+, HEK293 cells transiently transfected with the MC1R full-length cDNA (positive control); c1, c2, c3, primary HAoECs from ECACC, Lonza, and Promocell, respectively. (B) Intracellular cAMP concentrations were measured in confluent HAoECs after treatment with $\alpha$-MSH for 5 min, with or without the MC1R-selective $\alpha$-MSH antagonist 153 N-6. Results are shown as scatter dot plots with mean \pm SD ( $n=5-6$ per treatment group). Statistical significance of differences was assessed by one-way ANOVA $\left[\mathrm{F}_{(3,18)}=7.900, \mathrm{p}=0.0014\right]$ followed by Tukey's post-hoc test $\left({ }^{*} \mathrm{p}<0.05\right.$, ${ }^{* *} \mathrm{p}<0.01$ ). (C) Immunohistochemical detection of MC1R in a normal human aorta specimen $(10 \times)$ confirmed that HAoECs express the receptor in vivo. To control for staining specificity, we used secondary antibody alone (left), anti-MC1R antibody pre-adsorbed with the specific blocking peptide and secondary antibody (centre), and antiMC1R antibody with secondary antibody (right): only the latter showed an intense staining.

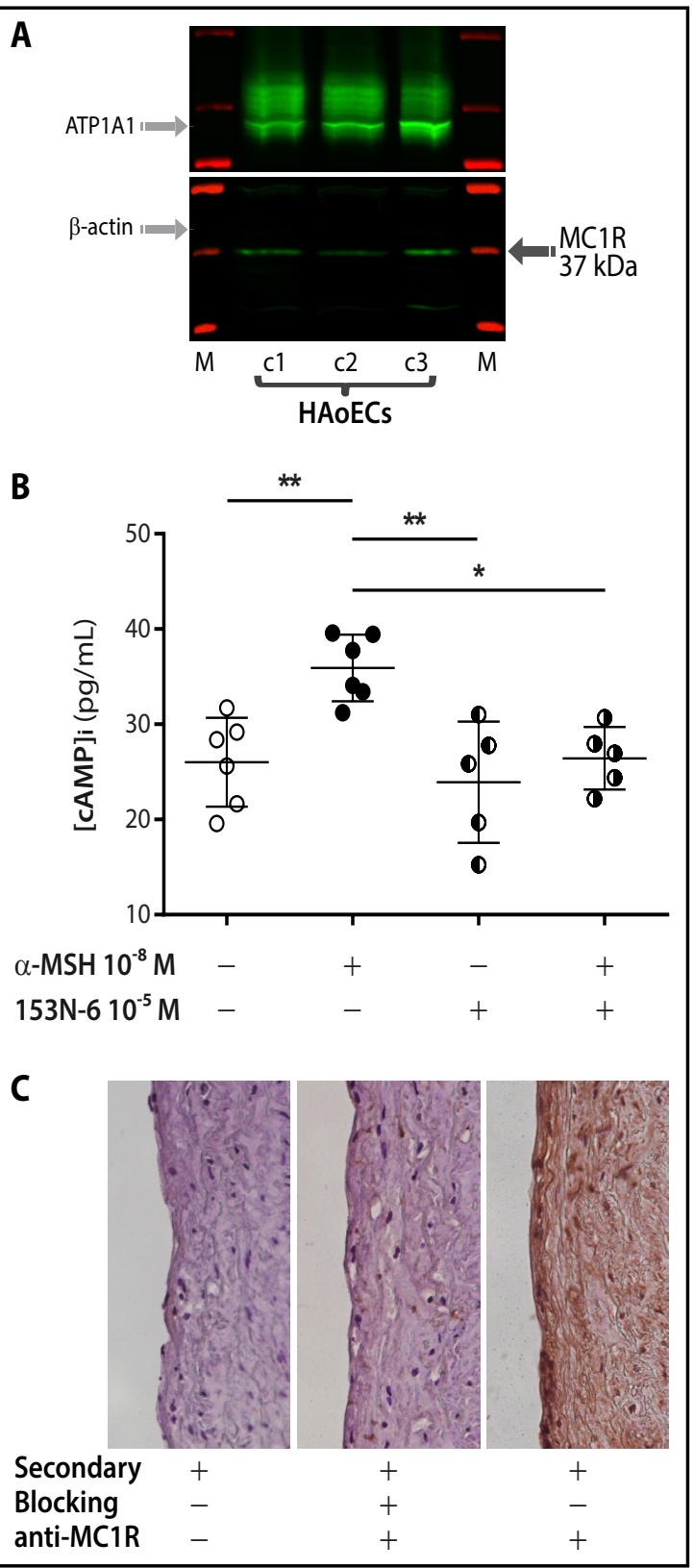


At variance with human dermal microvascular EC [10], POMC was undetectable in HAoECs and HCAECs. We detected the prohormone convertases (PCSK1, PCSK6, FURIN, and SCG5) [35] that process POMC into most of the derived peptides, but not PCSK2, which is needed to produce $\alpha$-MSH. Consistently, $\alpha$-MSH was undetectable in culture supernatants of all six macrovascular ECs (see online Supplementary Fig. S2).

To verify whether the three HAoECs express the MC1R protein, we performed immunoblot analysis. Specific immunoreactive bands, corresponding to the molecular weight of the canonical fully active receptor [36], were detected both in total cell lysates (see Supplementary Fig. S3A) and in membrane extracts (Fig. 1A and S3A), showing that the MC1R receptor was expressed on the plasma membrane of the HAoECs. Bands consistent with the MC1R dimeric forms were also detected (see Supplementary Fig. S1 and S3A). The level of expression of the MC1R monomer in total cell lysates was very similar among the three primary cell lines, whereas the HAoEC no. c3 appeared to express half the quantity of the

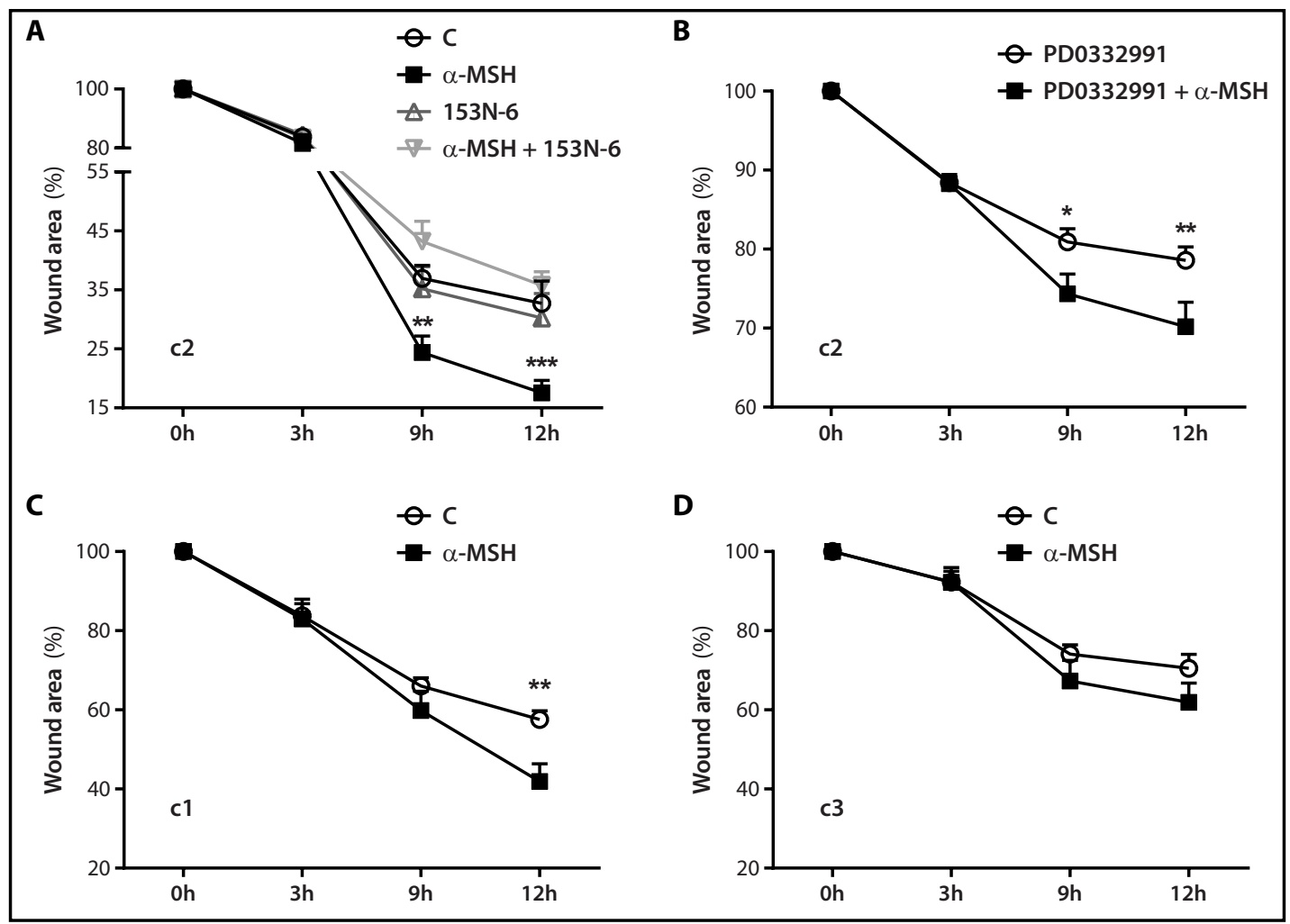

Fig. 2. MC1R activation enhances HAoEC migration. (A) After insert removal, HAoEC (no. c2) monolayers were treated with $\alpha$-MSH $10^{-8} \mathrm{M}$, with or without the MC1R-antagonist $153 \mathrm{~N}-610^{-5} \mathrm{M}$, and allowed to migrate for 3,9 , and $12 \mathrm{~h}$ : gap closure was quantified using DiR cell staining and near-infrared fluorescence scanning. Results are shown as mean \pm SEM $(n=6)$. Statistical significance of differences was assessed by two-way ANOVA $\left[\mathrm{F}_{(9,80)}=2.957, \mathrm{p}=0.0044\right.$, interaction time $\times$ treatment; $\mathrm{F}_{(3,80)}=10.85, \mathrm{p}<0.0001$, treatment effect] with Bonferroni post-hoc test [ ${ }^{* *} \mathrm{p}<0.01,{ }^{* * *} \mathrm{p}<0.001, \alpha-\mathrm{MSH}$ vs. medium alone (C)]. (B) Directional migration assay was repeated in the presence of the proliferation inhibitor PD0332991. Results are shown as mean \pm SEM $(n=10)$. Statistical significance of differences was assessed by two-way ANOVA $\left[F_{(3,72)}=\right.$ 3.018, $\mathrm{p}=0.0353$, for interaction; $\mathrm{F}_{(1,72)}=9.074, \mathrm{p}=0.0036$, treatment effect] with Bonferroni post-hoc test $\left({ }^{*} \mathrm{p}<0.05,{ }^{* *} \mathrm{p}<0.01\right)$. (C) The migration assay was repeated with HAoECs no. $c 1$, the other cell line bearing wild-type MC1R alleles. Results are shown as mean \pm SEM $(n=4)$. Statistical significance was assessed by two-way ANOVA $\left[\mathrm{F}_{(1,24)}=6.016, \mathrm{p}=0.0218\right.$, for treatment effect] with Bonferroni post-hoc test $(* * \mathrm{p}<0.01)$. (D) The migration assay was finally repeated with HAoECs no. $\mathrm{c} 3$, carrying a loss-of-function mutation in the MC1R gene. Results are shown as mean \pm SEM $(n=5)$. Two-way ANOVA with Bonferroni post-hoc test showed no statistically significant differences. 


\section{Cellular Physiology Cell Physiol Biochem 2019;52:1339-1360 \begin{tabular}{ll|l} 
and Biochemistry & $\begin{array}{l}\text { DOI: 10.33594/000000094 } \\
\text { Published online: 4 May 2019 }\end{array}$ & $\begin{array}{l}\text { C } 2019 \text { The Author(s). Published by } \\
\text { Cell Physiol Biochem Press GmbH\&Co. KG }\end{array}$
\end{tabular}

other two lines (see Supplementary Fig. S3B and S3C). Finally, the canonical monomeric form of the receptor was detected in all three HCAECs as well (see Supplementary Fig. S4).

Given that MC1R is a highly polymorphic gene and that many variants are known to affect its signal transduction [37], we sequenced the $M C 1 R$ ORF of the six primary human artery ECs to identify and exclude from subsequent functional analysis those cells with gene variants that may interfere with the cellular response to MC1R ligands. All the three HCAECs bear a variant allele, whereas two HAoECs did not present any polymorphism (see Supplementary Table S2). The variant alleles found have been associated with a decrease in cAMP production in response to $\alpha$-MSH stimulation [38-40]. For functional testing, we elected to use the HAoEC no. c2, which carried the wildtype receptor, due to its shorter doubling time.

We measured the changes in intracellular cAMP levels after treatment with $\alpha-\mathrm{MSH}$, to test whether confluent HAoECs express a functionally active MC1R. Indeed, 5-min stimulation with $\alpha$-MSH $10^{-8} \mathrm{M}$ induced a significant increase of intracellular cAMP in cells grown to confluence (Fig. 1B); cAMP elevation occurred in a concentrationdependent manner, showing a typical inverted U-shaped dose-response curve (see Supplementary Fig. S5A) [41, 42]. Coincubation with the receptor antagonist $153 \mathrm{~N}-6\left(10^{-5} \mathrm{M}\right)$ abolished the elevation of cAMP, indicating that MC1R is specifically activated by $\alpha$-MSH (Fig. 1B).

Finally, to test whether HAoECs express MC1R in vivo, we performed immunohistochemistry staining for the receptor in formaldehyde-fixed paraffin sections of a normal human aorta. We observed a positive staining of endothelial cells, confirming the in vitro observations (Fig. 1C).

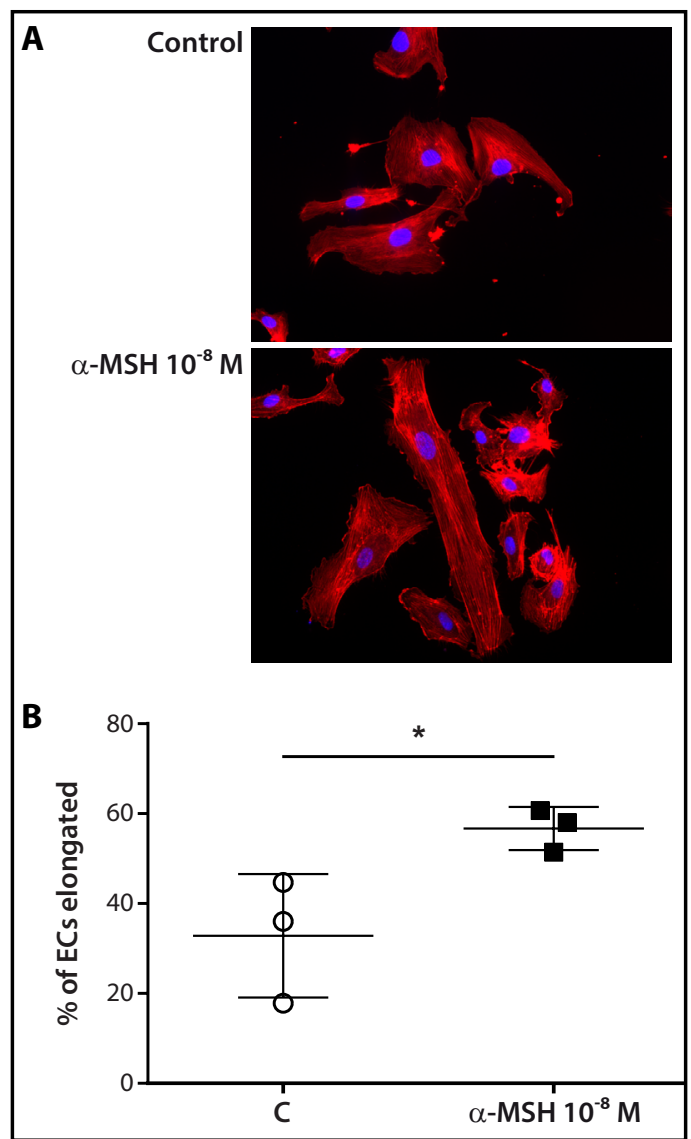

Fig. 3. MC1R activation enhances actin filament remodelling and cell elongation in migrating HAoECs. (A) Non-confluent ECs were stimulated with $\alpha$-MSH for $6 \mathrm{~h}$, then fixed and stained with TRITC-labelled phalloidin for actin filament visualization, using DAPI for nuclear counterstain $(40 \times)$. Aligned stress fibres and cellular elongation are pronounced in treated vs. untreated HAoECs. (B) Quantification of cell elongation. Cells with axial ratios $>3$ were counted in randomly selected fields and expressed as percentages of the total cells counted. Results are shown as scatter dot plots with mean \pm SD ( $n=3$ per group). Statistical significance of differences was assessed by twotailed unpaired t test $(* \mathrm{p}=0.0356)$.

\section{$\alpha$-MSH promotes migration of HAoEC via MC1R activation}

To determine whether MC1R activation has any influence on HAoEC migration and/ or proliferation, we used a directional cell migration assay. Stimulation with $\alpha$-MSH $10^{-8}$ $M$ enhanced HAoEC migration (Fig. 2A), and this effect too occurred in a concentrationdependent manner (see Supplementary Fig. S5B): in comparison with cells cultured in growth medium only, migration speed appeared to accelerate after $3 \mathrm{~h}$ of treatment and became significantly higher at 9 and $12 \mathrm{~h}$ in $\alpha$-MSH-treated cells. Consistently, concomitant use of $153 \mathrm{~N}-610^{-5} \mathrm{M}$ was able to abolish the pro-migratory effect of $\alpha$-MSH, whereas 


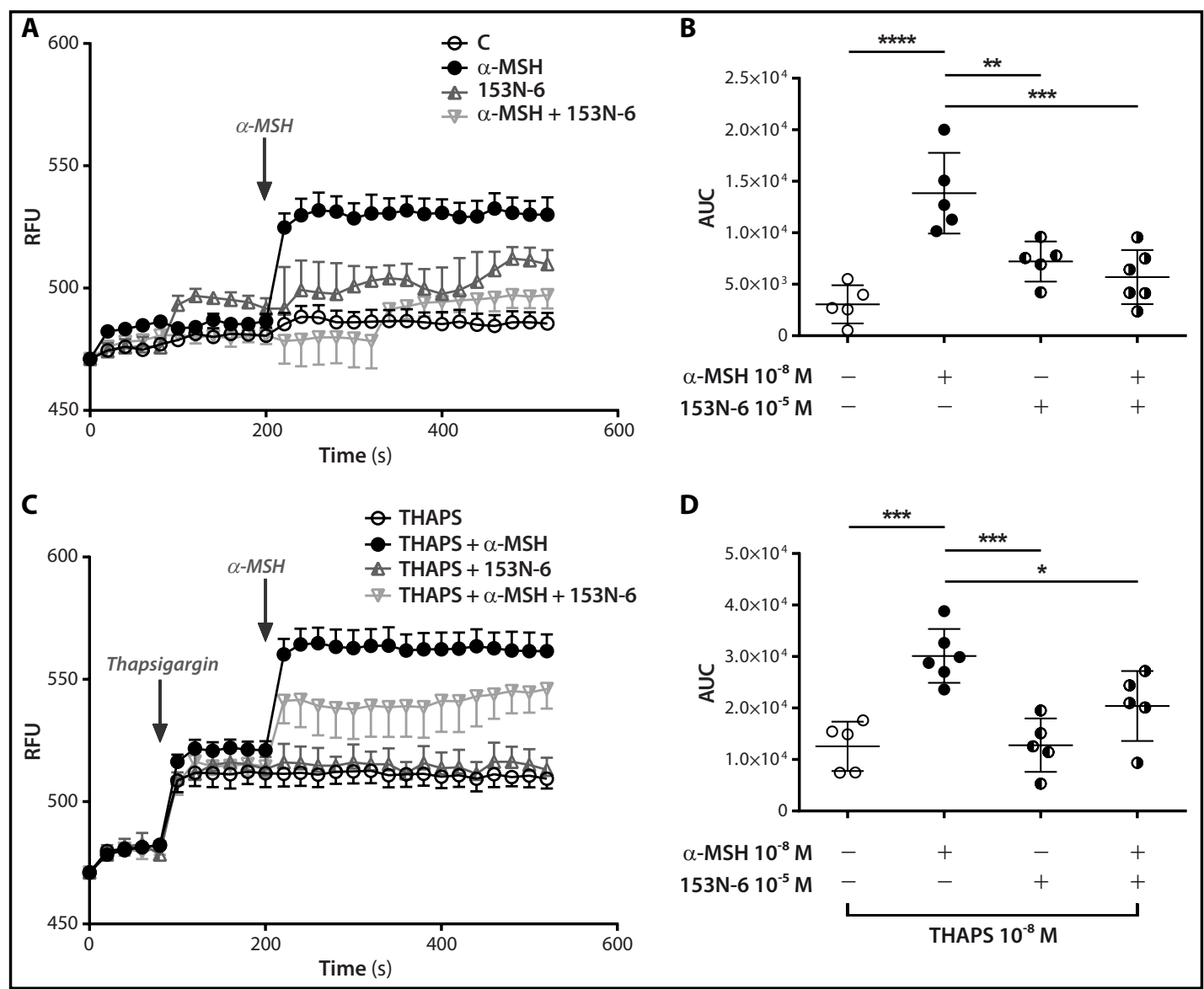

Fig. 4. MC1R activation increases intracellular calcium levels in migrating HAoECs. (A) Treatment with $\alpha$-MSH after insert removal in the cell migration assay induced a prompt increase in intracellular $\mathrm{Ca}^{2+}$ levels (as detected by Fluo-4 NW fluorescent calcium indicator), which was completely abolished by pre-treatment with the MC1R-antagonist 153N-6. (C) Rise of intracellular $\mathrm{Ca}^{2+}$ in response to the stimulus with $\alpha$-MSH was not prevented by prior stimulation with thapsigargin (THAPS). This was inhibited by pre-treating HAoECs with $153 \mathrm{~N}-6$. Arrows indicate thapsigargin or $\alpha$-MSH stimulation. Curves present the mean \pm SEM of $n=5-6$ independent experiments. RFU, relative fluorescence unit. (B, D) The areas under the curve (AUC) were used to compare $\alpha-\mathrm{MSH}$-induced effects with control treatments. Results are shown as scatter dot plots and mean \pm SD Statistical significance of differences was assessed by one-way ANOVA [(B) $\mathrm{F}_{(3,17)}=14.56, \mathrm{p}<0.0001$; (D) $\left.\mathrm{F}_{(3,16)}=12.50, \mathrm{p}=0.0001\right]$ with Tukey's post-hoc test $\left({ }^{*} \mathrm{p}<0.05,{ }^{* *} \mathrm{p}<0.01,{ }^{* * *} \mathrm{p}<0.001,{ }^{* * * *} \mathrm{p}<0.0001\right)$.

treatment with 153N-6 alone did not alter EC migration speed (also see representative images in Supplementary Fig. S6). Gap closure assays were then performed in the presence of the proliferation inhibitor PD0332991: as expected, blocking cell proliferation increased gap closure time, but the higher speed in $\alpha$-MSH-treated cells confirmed the enhancement in cell migration after MC1R activation, which was still significant at 9 and 12h (Fig. 2B and representative images in Supplementary Fig. S7A). To prove the generalizability of the promigratory effect of MC1R stimulation on macrovascular ECs, we showed that treatment with $\alpha$-MSH $10^{-8} \mathrm{M}$ significantly enhanced cell migration also in the other HAoEC line (no. c1) bearing the wild-type receptor, although at a lower speed (Fig. 2C and Supplementary Fig. S7B). Finally, to ascertain whether the observed responses were specifically dependent on the MC1R receptor subtype, we performed the same directional cell migration assay with the HAoEC line that was found to carry a loss-of-function allele in the MC1R gene (no. c3): interestingly, these cells showed an attenuated response to $\alpha$-MSH $\left(10^{-8} \mathrm{M}\right)$, with a slight non-significant acceleration in cell migration (Fig. 2D and Supplementary Fig. S7C). Of note, accelerated HAoEC migration upon activation of MC1R was confirmed in in vitro scratch 
wound healing assays (see Supplementary Fig. S8A and S8B): again, pre-treatment with the receptor antagonist $153 \mathrm{~N}-6\left(10^{-5} \mathrm{M}\right)$ abolished the effect. Conversely, there was no clear-cut effect on cell proliferation following MC1R activation, as documented by the number of Ki- 67 positive cells, which was not significantly different between treated and untreated HAoECs (see Supplementary Fig. S9A and S9B). MC1R expression did not significantly change over time during cell migration (not shown).

As cell migration is preceded by changes in cell morphology and actin filament remodelling, we evaluated whether these modifications occurred in HAoECs after $6 \mathrm{~h}$ from stimulation with $\alpha$-MSH $10^{-8} \mathrm{M}$. Indeed, we observed that MC1R activation through $\alpha-\mathrm{MSH}$ induced an accelerated shift from a "cobblestone", polygonal shape to an elongated shape in these ECs, with rearrangement of actin filaments (Fig. 3A). Phalloidin-TRITC staining showed formation of aligned stress fibres in $\alpha$-MSH-treated cells compared to untreated cells, whose actin filaments were mostly organized in short, unaligned stress fibres. HAoECs were then quantified for cell elongation, and cells stimulated with $\alpha$-MSH showed a significantly higher number of elongated cells in comparison with control cells (Fig. 3B).

Since MC1R may signal through either cAMP increase or intracellular elevation of free cytosolic $\mathrm{Ca}^{2+}[43]$, we tested which signal transduction pathway was active in the enhancement of the HAoEC migration. Stimulation with $\alpha$-MSH $10^{-8} \mathrm{M}$ did not lead to an increase of intracellular cAMP in migrating HAoECs compared to control cells (see Supplementary Fig. S10). On the contrary, MC1R activation resulted in a significant, rapid, and sustained increase in intracellular $\mathrm{Ca}^{2+}$ levels over the control, early after the removal of the insert in the cell migration assay (Fig. 4A). This rise was completely abolished when HAoECs were pre-treated with the $\alpha$-MSH antagonist $153 \mathrm{~N}-610^{-5} \mathrm{M}$, which in turn alone did not affect $\mathrm{Ca}^{2+}$ signalling. Comparisons of the AUCs confirmed that the $\alpha$-MSH-induced rise in $\mathrm{Ca}^{2+}$ levels was highly significant (Fig. 4B). Incubation with thapsigargin $\left(10^{-8} \mathrm{M}\right)$, a non-competitive inhibitor of sarco/endoplasmic reticulum $\mathrm{Ca}^{2+}$-ATPases (SERCAs) that causes a rapid raise of cytosolic $\mathrm{Ca}^{2+}$ by depleting endoplasmic reticulum stores [28], did not prevent a further significant rise of $\mathrm{Ca}^{2+}$ in response to a subsequent stimulus with $\alpha$-MSH (Fig. 4C and 4D). This was almost

Fig. 5. Calcium chelation or PLC inhibition prevent MC1R-mediated enhancement of HAoEC migration. (A) After insert removal, HAoEC (no. c2) monolayers were treated with $\alpha$-MSH $10^{-8} \mathrm{M}$, with or without pretreatment with the intracellular $\mathrm{Ca}^{2+}$ chelator BAPTAAM $10^{-5} \mathrm{M}$, and allowed to migrate for 3,9 , and $12 \mathrm{~h}$ : gap closure was quantified using DiR cell staining and near-infrared fluorescence scanning. Results are shown as mean \pm SEM ( $n=5-6$ per group). Statistical significance was assessed by two-way ANOVA $\left[\mathrm{F}_{(9,68)}\right.$ $=2.337, \mathrm{p}=0.0233$, interaction time $\times$ treatment; $\mathrm{F}_{(3,68)}=5.493, \mathrm{p}=0.0019$, treatment effect] with Bonferroni post-hoc test $\left[{ }^{*} \mathrm{p}<0.05,{ }^{* *} \mathrm{p}<0.01, \alpha-\mathrm{MSH}\right.$ vs. medium alone (C)]. (B) Directional migration assay was carried out also pre-treating cells with the PLC inhibitor U-73122. Results are shown as mean \pm SEM ( $\mathrm{n}=5-6)$. Statistical significance was assessed by two-way ANOVA $\left[\mathrm{F}_{(9,68)}=2.607, \mathrm{p}=0.0120\right.$, for interaction; $\mathrm{F}_{(3,68)}=7.102, \mathrm{p}=0.0003$, treatment effect] with Bonferroni post-hoc test $\left({ }^{*} \mathrm{p}<0.05\right)$.

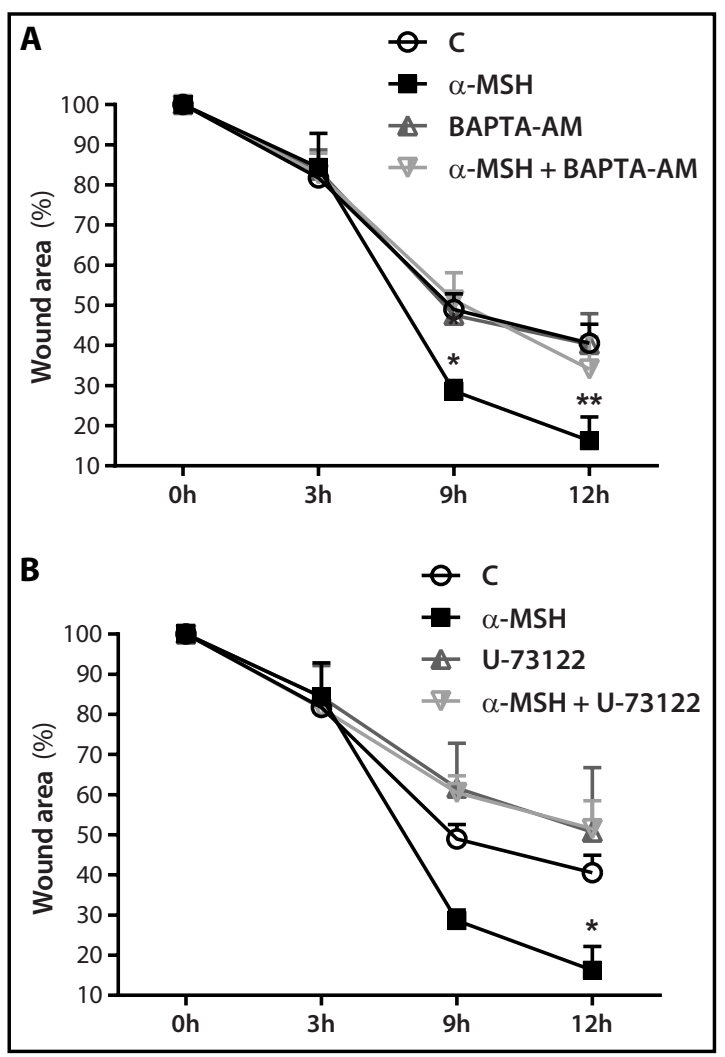


completely inhibited by pre-treating HAoECs with the $\alpha-\mathrm{MSH}$ antagonist $153 \mathrm{~N}-610^{-5} \mathrm{M}$. Intriguingly, $\alpha$-MSH $10^{-8} \mathrm{M}$ was able to induce $\mathrm{Ca}^{2+}$ mobilization also in confluent HAoECs, to levels comparable to those produced by thapsigargin (see Supplementary Fig. S11). In this case, thapsigargin almost completely hindered a further rise of $\mathrm{Ca}^{2+}$ in response to a subsequent stimulus with $\alpha$-MSH.

To further explore the $\mathrm{Ca}^{2+}$-dependency of the $\alpha$-MSH-induced cell migration, we repeated the directional cell migration assay in the presence of the membrane-permeant $\mathrm{Ca}^{2+}$ chelator BAPTA-AM, which is widely used as an intracellular $\mathrm{Ca}^{2+}$ sponge to control intracellular calcium ion concentration $\left(\left[\mathrm{Ca}^{2+}\right]\right.$ i) [44]. The pro-migratory effect of $\alpha$-MSH $10^{-8}$ $\mathrm{M}$, once more significant at 9 and $12 \mathrm{~h}$, was indeed completely inhibited by pre-treating HAoECs (no. c2) with BAPTA-AM $10^{-5} \mathrm{M}$ (Fig. $5 \mathrm{~A}$ ), confirming that $\mathrm{Ca}^{2+}$ mobilization via MC1R activation was involved in modulating cell migration. Finally, since $\alpha$-MSHstimulation of MC1R may induce $\left[\mathrm{Ca}^{2+}\right]_{i}$ elevation via activation of the $\mathrm{PLC}_{\beta}$ pathway $[45,46]$, which in turn has a key role in mediating EC functions and angiogenesis [47], we carried out the directional cell migration assay also in the presence of the $\mathrm{PLC}_{\beta}$ inhibitor U-73122. Again, the pro-migratory effect of $\alpha$-MSH $10^{-8} \mathrm{M}$ was inhibited by pretreating HAoECs with U-73122 $5 \times 10^{-5} \mathrm{M}$ (Fig. 5B), suggesting that $\alpha-\mathrm{MSH}$ evoked calcium mobilization/cell migration was dependent on the activation of $\mathrm{PLC}_{\beta}$.

Time-course expression analysis revealed several gene modules dynamically regulated by MC1R activation

To evaluate the effects of MC1R activation at the transcriptional level, we assessed genome-wide gene expression profiles at 0.5 , 3,6 , and $12 \mathrm{~h}$ after stimulation with $\alpha$-MSH $10^{-8} \mathrm{M} v$ s. control in HAoECs cultured in the directional cell migration assay. Applying stringent filtering parameters, we deemed expressed 18936 of the 47231 measured transcripts $(40 \%)$. Comparative time-course analysis, using the STEM algorithm [30], identified 637 genes whose expression consistently showed a median change $\geq 30 \%$ over time as an effect of stimulation with $\alpha$-MSH. These genes fitted 57 of the 625 possible model profiles computed by the

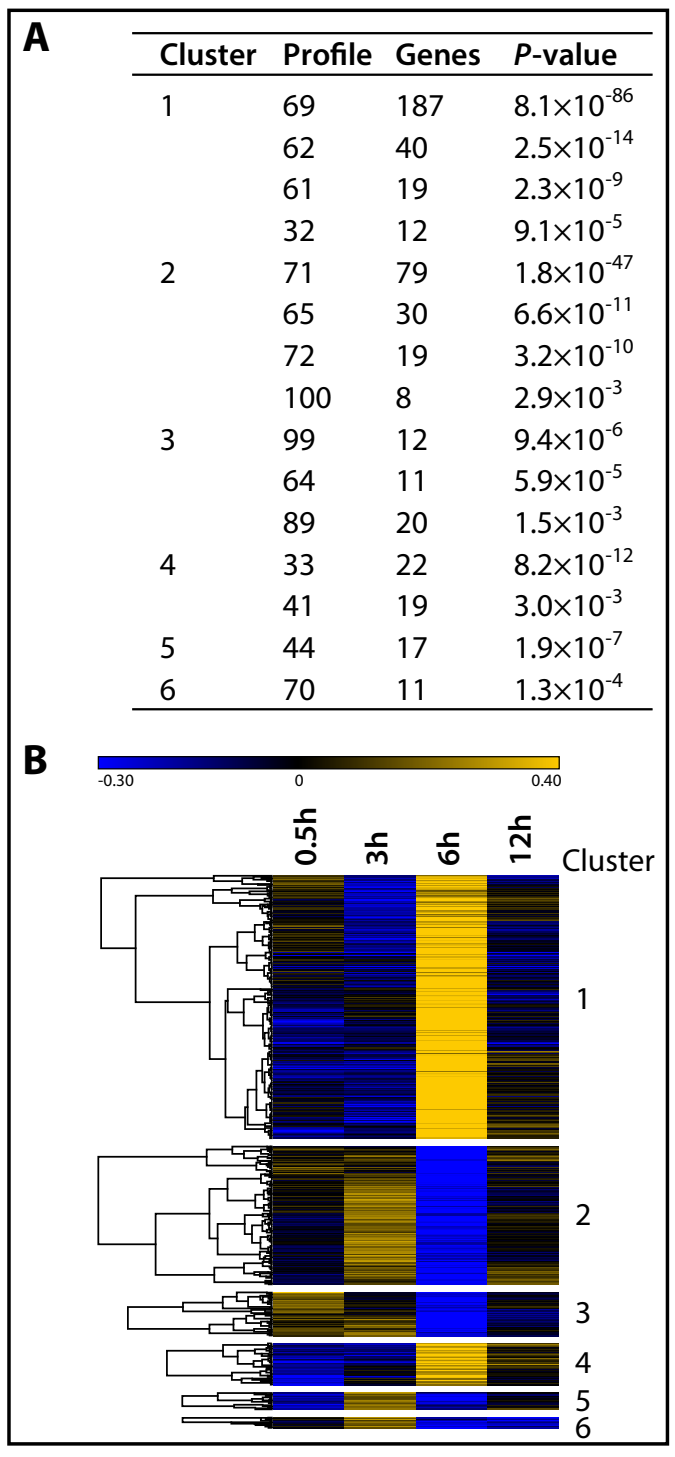

Fig. 6. Significant temporal expression profiles of genes modulated by MC1R activation in migrating HAoECs. (A) List of significantly enriched temporal expression patterns identified by STEM analysis. Expression profiles are grouped into six clusters based on their similarity $(r \geq 0.7)$ and ordered by $p$-value significance within each cluster profile. The number of genes belonging to a profile is reported. (B) Heatmap depicting temporal expression of genes within each cluster. Genes hierarchically clustered into 6 groups using one minus Pearson correlation distance and the average linkage method. Data are the average $\log _{2}$ gene expression ratio of $\alpha$-MSH stimulated cells to non-stimulated cells ( $\mathrm{n}=3$ independent experiments, with two technical replicates each). Normalized expression ratios are shown as a gradient colour ranging from lower (blue) to higher (gold) values. 


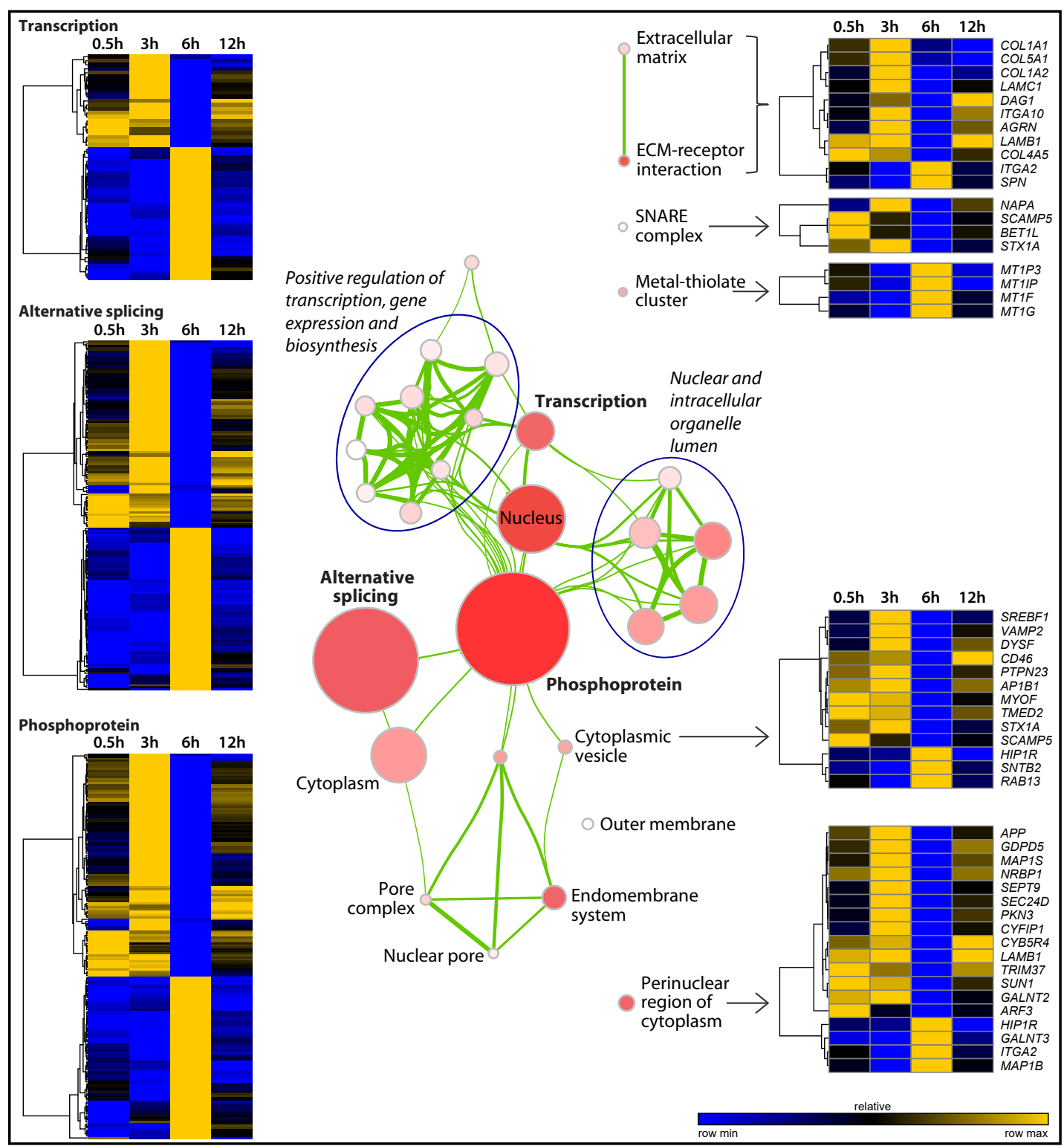

Fig. 7. Modules of co-regulated genes in migrating HAoECs upon MC1R activation. The enrichment map of modulated genes was drawn as a network of the most significant functionally annotated gene sets $(\mathrm{p}<0.01$ and Benjamini FDR<0.20). Nodes represent gene sets. Node colour intensity is relative to enrichment significance, from lower (light) to higher (dark red). Node size is proportional to the gene set size. Gene sets are connected by green edges based on their similarity. Edge thickness measures the degree of the overlap between two gene sets (using a cut-off of the Jaccard plus Overlap combined coefficient $=0.375$ ). Clusters of tightly, functionally related gene sets are circled and assigned an overall label. Heat maps of temporal expression patterns of relevant gene sets and pathways are displayed. Hierarchical clustering of genes was performed using one minus Pearson correlation distance and the average linkage method. Row normalized expression values are shown as a gradient colour ranging from lower (blue) to higher (gold) values.

clustering algorithm (see Supplementary Fig. S12). Five hundred and six transcripts were associated with 15 distinct temporal profiles that showed a statistically significant enriched number of genes at a FDR $<0.05$ (see Supplementary Fig. S12 and Table S3a). The remaining 131 genes were associated with model profiles that had a FDR $>0.05$ (see Supplementary Table S3b) and, thus, were deemed as potentially arising from noise by random chance and excluded from subsequent analysis. Interestingly, we did not observe any significant change 
in $M C 1 R$ expression level in migrating ECs after stimulation with $\alpha$-MSH at any time point. The 15 significant temporal profiles of differential expression were grouped, based on their similarity by a correlation coefficient $\geq 0.7$, to form 6 different clusters (Fig. 6A and 6B). Overall, genes belonging to clusters 1 and 4 showed a marked increase in expression at $6 \mathrm{~h}$ in treated vs. untreated cells, whereas genes in clusters 2 and 3 displayed a marked decrease at the same time point; conversely, genes in clusters 5 and 6 appeared to be upregulated at $3 \mathrm{~h}$.

Time-course expression data of control and $\alpha-\mathrm{MSH}$ stimulated cells at 3 and $6 \mathrm{~h}$ were validated using PCR-based arrays profiling key genes involved in wound healing. Forty-eight genes were detected by both microarray and real-time PCR (see Supplementary Fig. S13), with a strong positive correlation between their average signal intensities $(r \geq 0.8, \mathrm{p}<0.0001$ for all pairwise correlations).

To uncover the biological meaning beneath these transcriptional effects, we performed a functional enrichment analysis of the 506 regulated genes (see Supplementary Table S4). Forty-four terms were significantly enriched at $\mathrm{p}<0.01$ and FDR $<0.20$ and were used to draw a network to visually interpret biological data (Fig. 7). The most significant gene sets included the phosphoprotein class (FDR $<0.00002)$, the endomembrane system (FDR $<0.015$ ), and the ECM-receptor interaction (FDR<0.025). Notably, 197 of the 506 regulated genes encode for phosphoproteins, 188 produce variant proteins by alternative splicing, and 65 are transcription factors or regulators. Importantly, 11 genes, i.e. AGRN, COL1A1, COL1A2, COL4A5, COL5A1, DAG1, ITGA2, ITGA10, LAMB1, LAMC1, and SPN, belonged to either the ECMreceptor interaction pathway or the extracellular matrix cellular component.

To further analyse gene expression changes in a structured fashion, functionally enrichment analysis was performed associating annotated gene sets with the 6 different

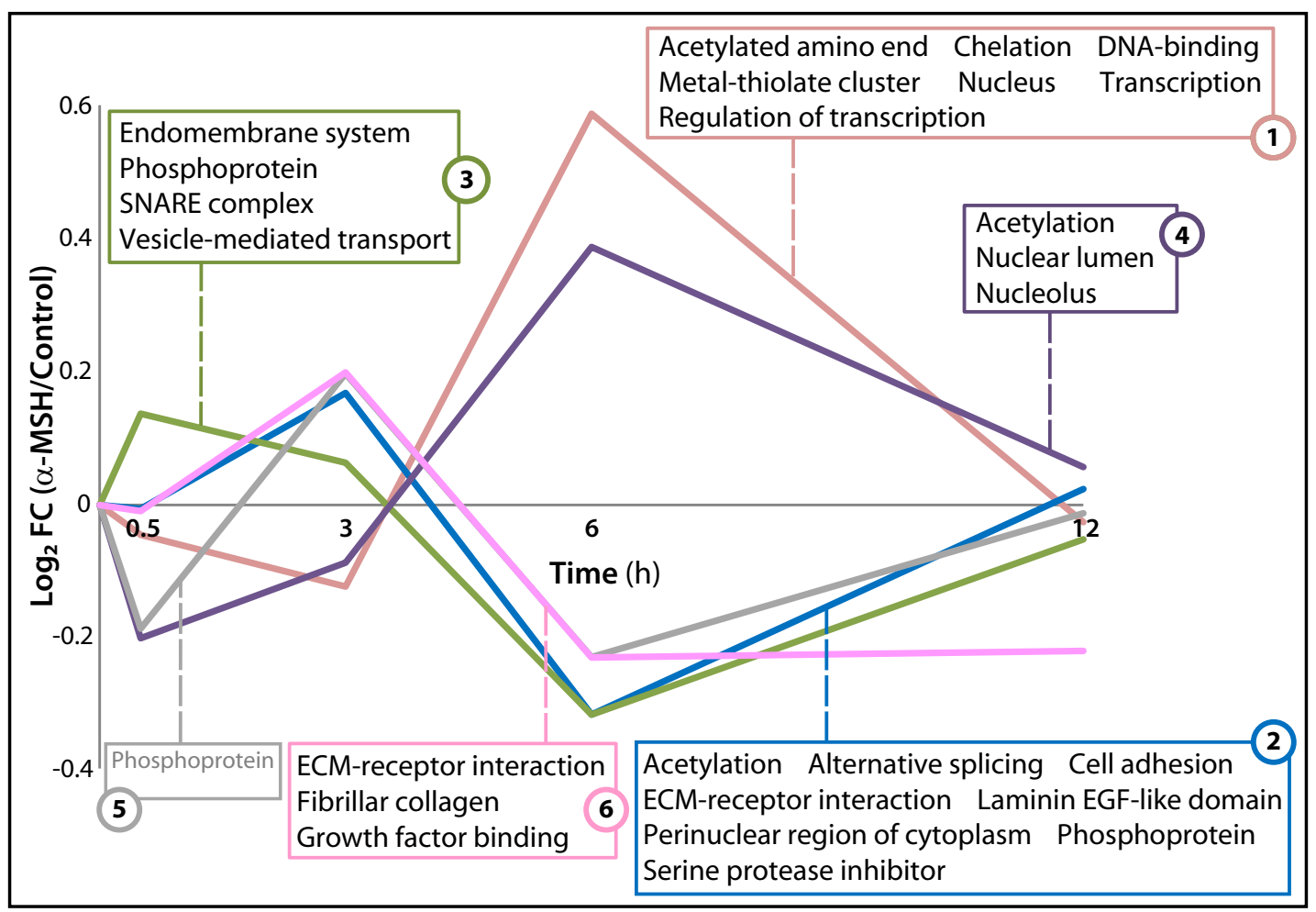

Fig. 8. Time-course gene cluster profiles. The 6 different expression profiles represent time-dependent dynamic gene modulation as the mean of significant temporal profiles grouped on the basis of their similarity. Each cluster profile is associated with gene sets and pathways (coloured rectangles) significant at the enrichment analysis. For cluster 5 , a gene set with a nominal $p<0.05$ is indicated. On the $y$-axes is the $\log _{2}$ mean fold change (FC) relative to control cells, i.e. the $\log _{2}$ gene expression ratio of $\alpha$-MSH stimulated cells to non-stimulated cells; on the $\mathrm{x}$-axis is the experimental time scale (hours). 
clusters of temporal profiles (see Supplementary Table S5). Co-expressed gene subsets were visualized as temporal clustered profiles sharing functional annotations (Fig. 8). Coupling time-course gene expression analysis to enrichment analysis allowed identifying significantly regulated genes that have never been associated with MC1R signalling before, including genes involved in ECM-receptor interaction, vesicle-mediated transport, SNARE protein complex formation, and metal ion binding through metal-thiolate cluster structures (metallothioneins, MTs).

\section{Discussion}

In this work, we provide the first formal demonstration that human artery ECs express constitutively a functional MC1R and present evidence that activation of this melanocortin receptor drives faster EC migration and wound closure. Besides, time-course gene expression analysis allowed us identifying downstream molecular pathways associated with the enhanced cell motility following $\alpha$-MSH engagement of the MC1R. This observation adds to the known functions of the melanocortin system, which is known to regulate homeostasis and possess cellular and tissue protective effects [2]. It is worth mentioning that our data are apparently in contrast with the notion that $\alpha$-MSH inhibits migration of several cell types, such as immune/inflammatory [6, 7] or melanoma cells [48]: our findings pinpoint that the spectrum of action of MC1R signalling is wider and more diversified than previously thought, possibly depending on the cell type and pathophysiological context. Similarly, $\alpha$-MSH binding to the MC1R stimulates or inhibits the proliferation respectively of cultured human melanocytes [49] and mesothelioma cell lines [50].

We detected both the MC1R mRNA and protein and demonstrated that it was functionally active, since resting confluent HAoECs were able to produce cAMP in response to exogenous $\alpha-\mathrm{MSH}$. No other component of the melanocortin system was detectable in HAoECs. Consistently, we did not detect $\alpha$-MSH in cell culture supernatants. Thus, HAoECs are not a source of melanocortin peptides, but may be targeted by endocrine secretion (by the pituitary gland) or paracrine release of endogenous agonists (e.g. by immune cells at injured sites) [6, 7]. These findings represent a peculiar difference between macrovascular and microvascular ECs, as it has been reported that HDMECs express POMC and release melanocortin peptides upon stimulation [10]. In addition, unlike the microvascular ECs [12], $M C 1 R$ expression did not significantly changed upon exposure of the HAoECs to $\alpha$-MSH. This finding suggests that in macrovascular ECs MC1R expression levels are not influenced by pathways that depend on its activation and that HAoEC migration is not dependent on $M C 1 R$ upregulation.

These results underline that artery ECs present cell type-restricted gene expression, which may account for a specific physiological function for MC1R, other than antiinflammatory actions. This hypothesis led us to investigate whether MC1R activation could affect macrovascular EC migration after injury. EC migration is a fundamental process primed by damage and involved in vascular homeostasis and repair. Our data revealed that MC1R activation by $\alpha$-MSH increased the rate of HAoEC migration, both in gap-closure and in injuryinduced wound-healing assays, without significantly affecting cell proliferation. This effect was specifically induced by MC1R activation, since the $153 \mathrm{~N}-6$ peptide antagonist at MC1R [25] abolished the $\alpha$-MSH-driven migration of HAoECs, reverting it to the same rate of control cells. Consistently, ECs carrying a loss-of-function mutation in the MC1R gene did not show a significant acceleration in cell motility upon challenge with $\alpha-\mathrm{MSH}$. In seeming contrast to what we observed, it has been recently reported that $\alpha$-MSH inhibits in vitro migration of HUVECs [51]; but this reinforces our idea that the pro-migratory effect of $\alpha$-MSH via MC1R activation is restricted to arterial ECs of the macrovasculature. Accordingly, we observed a prompt elevation of intracellular free $\mathrm{Ca}^{2+}$ after $\alpha$-MSH stimulation in migrating cells, but not of cAMP, suggesting that MC1R activation enhances EC migration through the $\mathrm{Ca}^{2+}$ signalling cascade. Indeed, artificial intracellular calcium buffering by pre-treating cells with the cell- 
permeant $\mathrm{Ca}^{2+}$ chelator BAPTA-AM completely abolished the $\alpha$-MSH-evoked acceleration in EC migration. Increase of calcium levels in the cytosol is an evolutionary conserved signal involved in the regulation of EC motility. $\mathrm{Ca}^{2+}$ mobilization can both stabilize and weaken cell-ECM interactions responsible for the asymmetry between cell front and rear adhesions, which finally results in cellular directed movement [52-54].

Intriguingly, our experiments showed that HAoEC MC1R might signal by increasing cAMP and/or intracellular $\mathrm{Ca}_{2}{ }^{+}$depending on the cellular state: resting confluent HAoECs responded to MC1R engagement with $\alpha$-MSH through cAMP and $\left[\mathrm{Ca}^{2+}\right]_{i}$ increase, while migrating cells responded through $\mathrm{Ca}^{2+}$ mobilization without any cAMP increase. To date, only a few reports support an involvement of calcium as a second messenger in MC1R signalling, besides cAMP. $\mathrm{Ca}^{2+}$ responses has been reported in HEK 293 cells transfected with mouse Mc1r [55] and in human melanoma cell lines [48], keratinocytes [56], and basophils [57] expressing MC1R. No elevation in cAMP was detected in keratinocytes and basophils in response to $\alpha$-MSH $[56,57]$, whereas in melanoma cells and keratinocytes intracellular $\mathrm{Ca}^{2+}$ release was observed only in the presence of a pharmacological adenosine agonist that inhibits the cAMP pathway $[48,56]$. Conversely, our findings provide evidence that MC1R couples to both cAMP and $\mathrm{Ca}^{2+}$ signalling systems in HAoECs and suggest that different functional states may direct alternative signalling pathways in macrovascular ECs. This is remarkable: MC1R appears to be one of those GPCRs that may simultaneously couple to distinct unrelated G-proteins and alternatively activate multiple intracellular effectors [58] depending on cell type, physiological condition, and the availability of G-protein $\left(G_{\alpha s}\right.$ or $\left.G_{\alpha q}\right)$ adaptors [59]. Of note, in the MCR family, alternative G-protein coupling has been reported for MC4R [60].

In HEK 293 cells transfected with $M c 1 r$, complete depletion of intracellular $\mathrm{Ca}^{2+}$ stores following pre-treatment with thapsigargin $10^{-8} \mathrm{M}$ abolished a further rise in $\left[\mathrm{Ca}^{2+}\right]$ in response to $\alpha$-MSH, suggesting that the thapsigargin-sensitive endoplasmic reticulum $\mathrm{Ca}^{2+}$ stores were the source of $\left[\mathrm{Ca}^{2+}\right]_{\mathrm{i}}$ increase [55]. This appears to be the case also for confluent HAoECs. On the contrary, in migrating HAoECs this concentration of thapsigargin was not able to inhibit a further rise in $\left[\mathrm{Ca}^{2+}\right]_{\mathrm{i}}$ in response to $\alpha-\mathrm{MSH}$, suggesting that either SERCAs were only partially inhibited or $\alpha$-MSH may increase intracellular $\mathrm{Ca}^{2+}$ from other sources. Indeed, angiogenic factors induce cytosolic calcium rises through either entry from extracellular space, by opening $\mathrm{Ca}^{2+}$ permeable channels in the plasma membrane, or release from intracellular organelles [61]. On the other hand, it has been shown that thapsigargin $1 \mu \mathrm{M}$ may activate $\mathrm{Ca}^{2+}$ entry both by store-dependent and store-independent pathways in the HUVEC line EA.hy926 [62]. Here, we provide mechanistic insight showing that $\alpha$-MSHinduced intracellular $\mathrm{Ca}^{2+}$ mobilization and accelerated migration are dependent on the activation of the PLC signalling pathway: in fact, the blockage of PLC by the specific inhibitor U-73122 completely abrogated the increase in HAoEC migration rate via MC1R activation. GPCR-mediated PLC $_{\beta}$ activation cleaves membrane-bound phosphatidylinositol 4, 5 bisphosphate $\left(\mathrm{PIP}_{2}\right)$ into inositol $(1,4,5)$ trisphosphate $\left(\mathrm{IP}_{3}\right)$ and diacylglycerol; $\mathrm{IP}_{3}$ binding to $\mathrm{IP}_{3}$ receptor $\left(\mathrm{IP}_{3} \mathrm{R}\right.$ ) channels promotes $\mathrm{Ca}^{2+}$ release from the endoplasmic reticulum [63]. Consistent with our observation, MC1R has been recently shown to transduce through the $\mathrm{PIP}_{2}-\mathrm{PLC}_{\beta}$ pathway in sebocytes [45] and B16-F10 melanoma cells [46].

Time-series genome-wide gene-expression analysis on migrating HAoECs showed that large gene sets were significantly affected by $\alpha$-MSH treatment: i.e., genes involved in the regulation of RNA transcription, encoding for proteins for which isoforms exist due to premRNA splicing events (alternative splicing), and genes belonging to the phosphoprotein category. This indicates that MC1R activation has a wide influence on pathways playing a prominent role in regulating cellular activity. MC1R activation also modulated genes associated with the endomembrane system and intracellular organelle lumen, suggesting a role in controlling cellular trafficking and molecule mobilization. Remarkably, MC1R engagement with $\alpha$-MSH affected the ECM-receptor interaction pathway, which is known to be critical for the directional haptotactic EC migration [64]. Conversely, MC1R activation did not affect the expression of cell cycle-related genes, which was consistent with the apparent 
lack of effect on HAoEC proliferation in the gap closure assay. Our findings suggest that the regulation of the ECM components, i.e. collagens and laminins, and of their receptors, i.e. integrins and dystroglycans, through MC1R may drive higher HAoEC motility. $\alpha$-MSH appears to boost HAoEC migration regulating the interaction between the cellular receptor integrins (ITGA2 and ITGA10) and DAG1 to their ECM counterpart collagens (COL1A1, COL1A2, COL4A5, and COL5A1), laminins (LAMB1 and LAMC1) and AGRN. The directed motility of ECs is strictly dependent on cell adhesion to ECM $[64,65]$. Integrins and interstitial collagen mediate haptotactic cell migration, which is of primary importance in driving EC migration during large vessel repair $[21,66]$. Furthermore, time-course analysis evidenced that 9 genes of the ECM-receptor interaction pathway had a similar temporal expression profile, with a peak induction at $3 \mathrm{~h}$ followed by a reversion at $6 \mathrm{~h}$, suggesting that common factor(s) may control their co-expression. Conversely, ITGA2 and $S P N$ showed a specular temporal profile, with a later peak expression at $6 \mathrm{~h}$, which is suggestive of a sequential upregulation of ECMreceptor interaction genes [67]. Remarkably, we showed that $\alpha$-MSH enhances EC migration along with actin filament remodelling and changes in cell architecture. Binding of integrins to type-I collagen suppresses cAMP production and the activity of cAMP-dependent protein kinase A: consequently, actin polymerization is induced, contributing to the formation of stress fibres and to EC contractility, which finally generates the directional movement [68]. This is consistent with the idea that the fine-tuning of integrins and their binding molecules promoted by MC1R activation plays a key role in conditioning HAoEC migration rate. In addition, MC1R stimulation induced an early upregulation of SNARE proteins (which mediate vesicle-membrane fusion) and cytoplasmic vesicle genes, followed by a later overexpression of metal-binding proteins. Importantly, trafficking and delivery/fusion vesicle proteins are essential for the regulation of front-rear polarity during directional cell migration [69, 70]. Likewise, MTs enhance EC motility [71] and angiogenesis [72] through transcriptional regulation of various vascular growth-factors, and their modulation drove suppression of reactive oxygen species production in ECs exposed to elevated laminar shear stress [73]. Such a pattern of temporal dynamics in gene expression (i.e. ECM-receptor interaction, SNARE, or MT genes) is expected as an "impulse response" to a transient signal, namely a single pulse of $\alpha$-MSH [74]. This typical oscillating wave of co-expressed genes may reflect a highly ordered temporal organization in gene transcription, which ultimately results in the subsequent, coordinated translation into the corresponding effector proteins that drive the $\alpha$-MSH-mediated increase in EC migration speed. Consistently, the peaks in gene expression were followed at 6-12 hours by a transition to the steady state.

In summary, MC1R activation via $\alpha$-MSH appears to accelerate directional HAoEC migration through the following steps: $(a)$ binding of melanocortin hormones to MC1R induces $(b)$ an increase in cytosolic $\mathrm{Ca}^{2+}$ while preventing a rise in cAMP biosynthesis, through a putative alternative G-protein coupling and PLC-pathway activation, and subsequently $(c)$ the coordinate modulation of genes of the ECM-receptor interaction, vesicle- and SNAREmediated trafficking pathways, and metal sensing proteins, $(d)$ possibly regulating the cell front-rear polarity. These responses reflect an unrecognized protective function of the melanocortin system, which is fostered by previously unreported $\alpha$-MSH-activated, MC1Rmediated signalling and molecular pathways.

MC1R tonic signalling and pro-migratory action may be relevant for the homeostatic functions of the arterial endothelium. The endothelium monolayer lining in the luminal side of blood vessels plays a pivotal role in the regulation of the haemostatic balance, prevention of vascular inflammation, and protection against vascular injury [75]. Normal ECs express a number of inhibitors of platelet and leukocyte activation, vasodilators, and anticoagulant and procoagulant molecules. Damage to these cells is associated with a shift in the haemostatic balance to the procoagulant side [15], loss of protective molecules and expression of adhesive, inflammatory and mitogenic factors, leading to the development of thrombosis, pathologic remodelling, and atherosclerosis [75]. Endothelial dysfunction is characterized by an imbalance between procoagulant and anticoagulant mediators and regenerated arterial endothelium may be functionally incompetent with reduced expression of antithrombotic 
molecules $[15,76]$. EC migration is a key event in wound healing and tissue regeneration, including reendothelialisation after stent implantation [76]. Remarkably, MC1R activation in migrating HAoEC did not alter the balance between pro- and anticoagulant genes, i.e. expression of procoagulant (such as $V W F, F 2 R$, and $F 3$ ) and anticoagulant genes (THBD, $H S P G, E P C R$, and TFPI) was not affected by $\alpha-\mathrm{MSH}$. Thus, MC1 R activation may have beneficial effects both ameliorating HAoEC motility properties and maintaining the equilibrium between pro- and anticoagulant signals.

\section{Conclusion}

Our work broadens the knowledge on MCR regulatory roles and supports the concept of a novel function for peripherally expressed MC1R, whose signalling may participate in preventing/healing of artery endothelial dysfunction, vascular repair, and reendothelialisation. Endothelial artery MC1R could represent a target for original therapeutic strategies aimed at preventing/repairing endothelial injury in a variety of cardiovascular pathological conditions associated with endothelial denudation [20].

\section{Acknowledgements}

We thank Dr Chiara Speroni for help and excellent technical assistance. We thank Fondazione Banca di Treviso ONLUS for kindly providing us with the human aorta specimen. This study was supported by Institutional Research Funds (Italian Ministry of Health, Funds 5\% 2009-11; to G.I.C.).

\section{Disclosure Statement}

The authors declare no conflict of interests.

\section{References}

- 1 Dores RM, Londraville RL, Prokop J, Davis P, Dewey N, Lesinski N: Molecular evolution of GPCRs: Melanocortin/melanocortin receptors. J Mol Endocrinol 2014;52:T29-T42.

- 2 Cone RD: Studies on the physiological functions of the melanocortin system. Endocr Rev 2006;27:736-749.

- 3 Rodrigues AR, Almeida H, Gouveia AM: Intracellular signaling mechanisms of the melanocortin receptors: current state of the art. Cell Mol Life Sci 2015;72:1331-1345.

- 4 Catania A, Gatti S, Colombo G, Lipton JM: Alpha-melanocyte stimulating hormone in modulation of inflammatory reactions. Pediatr Endocrinol Rev 2003;1:101-108.

5 Colombo G, Gatti S, Turcatti F, Sordi A, Fassati LR, Bonino F, Lipton JM, Catania A: Gene expression profiling reveals multiple protective influences of the peptide alpha-melanocyte-stimulating hormone in experimental heart transplantation. J Immunol 2005;175:3391-3401.

6 Brzoska T, Luger TA, Maaser C, Abels C, Bohm M: Alpha-melanocyte-stimulating hormone and related tripeptides: biochemistry, antiinflammatory and protective effects in vitro and in vivo, and future perspectives for the treatment of immune-mediated inflammatory diseases. Endocr Rev 2008;29:581-602.

7 Catania A, Gatti S, Colombo G, Lipton JM: Targeting melanocortin receptors as a novel strategy to control inflammation. Pharmacol Rev 2004;56:1-29.

8 Haycock JW, Rowe SJ, Cartledge S, Wyatt A, Ghanem G, Morandini R, Rennie IG, MacNeil S: Alphamelanocyte-stimulating hormone reduces impact of proinflammatory cytokine and peroxide-generated oxidative stress on keratinocyte and melanoma cell lines. J Biol Chem 2000;275:15629-15636. 


\section{Cellular Physiology Cell Physiol Biochem 2019;52:1339-1360 \begin{tabular}{l|l} 
and Biachemistry 2019 The Author(s). Published by \\
\hline DOI 10.33594/000000094 & C
\end{tabular} and BIOChemistry Published online: 4 May $2019 \quad$ Cell Physiol Biochem Press GmbH\&Co. KG \\ Saporiti et al.: MC1R Enhances Endothelial Cell Migration}

9 Zhang L, Dong L, Liu X, Jiang Y, Zhang X, Li X, Zhang Y: alpha-Melanocyte-stimulating hormone protects retinal vascular endothelial cells from oxidative stress and apoptosis in a rat model of diabetes. PLoS One 2014;9:e93433.

10 Scholzen TE, Brzoska T, Kalden DH, Hartmeyer M, Fastrich M, Luger TA, Armstrong CA, Ansel JC: Expression of functional melanocortin receptors and proopiomelanocortin peptides by human dermal microvascular endothelial cells. Ann N Y Acad Sci 1999;885:239-253.

11 de Angelis E, Sahm UG, Ahmed AR, Olivier GW, Notarianni LJ, Branch SK, Moss SH, Pouton CW: Identification of a melanocortin receptor expressed by murine brain microvascular endothelial cells in culture. Microvasc Res 1995;50:25-34.

12 Hartmeyer M, Scholzen T, Becher E, Bhardwaj RS, Schwarz T, Luger TA: Human dermal microvascular endothelial cells express the melanocortin receptor type 1 and produce increased levels of IL-8 upon stimulation with alpha-melanocyte-stimulating hormone. J Immunol 1997;159:1930-1937.

13 Rinne P, Nordlund W, Heinonen I, Penttinen AM, Saraste A, Ruohonen ST, Makela S, Vahatalo L, Kaipio K, Cai M, Hruby VJ, Ruohonen S, Savontaus E: alpha-Melanocyte-stimulating hormone regulates vascular NO availability and protects against endothelial dysfunction. Cardiovasc Res 2013;97:360-368.

- 14 Aird WC: Phenotypic heterogeneity of the endothelium: I. Structure, function, and mechanisms. Circ Res 2007;100:158-173.

- 15 Aird WC: Endothelium and haemostasis. Hamostaseologie 2015;35:11-16.

- 16 Chi JT, Chang HY, Haraldsen G, Jahnsen FL, Troyanskaya OG, Chang DS, Wang Z, Rockson SG, van de Rijn M, Botstein D, Brown PO: Endothelial cell diversity revealed by global expression profiling. Proc Natl Acad Sci U S A 2003;100:10623-10628.

- 17 Bhasin M, Yuan L, Keskin DB, Otu HH, Libermann TA, Oettgen P: Bioinformatic identification and characterization of human endothelial cell-restricted genes. BMC Genomics 2010;11:342.

18 Cines DB, Pollak ES, Buck CA, Loscalzo J, Zimmerman GA, McEver RP, Pober JS, Wick TM, Konkle BA, Schwartz BS, Barnathan ES, McCrae KR, Hug BA, Schmidt AM, Stern DM: Endothelial cells in physiology and in the pathophysiology of vascular disorders. Blood 1998;91:3527-3561.

19 Rinne P, Silvola JM, Hellberg S, Stahle M, Liljenback H, Salomaki H, Koskinen E, Nuutinen S, Saukko P, Knuuti J, Saraste A, Roivainen A, Savontaus E: Pharmacological activation of the melanocortin system limits plaque inflammation and ameliorates vascular dysfunction in atherosclerotic mice. Arterioscler Thromb Vasc Biol 2014;34:1346-1354.

20 Otsuka F, Finn AV, Yazdani SK, Nakano M, Kolodgie FD, Virmani R: The importance of the endothelium in atherothrombosis and coronary stenting. Nat Rev Cardiol 2012;9:439-453.

- 21 Michaelis UR: Mechanisms of endothelial cell migration. Cell Mol Life Sci 2014;71:4131-4148.

- 22 Herraiz C, Olivares C, Castejon-Grinan M, Abrisqueta M, Jimenez-Cervantes C, Garcia-Borron JC: Functional Characterization of MC1R-TUBB3 Intergenic Splice Variants of the Human Melanocortin 1 Receptor. PLoS One 2015; 10:e0144757.

23 Andersen CL, Jensen JL, Orntoft TF: Normalization of real-time quantitative reverse transcription-PCR data: a model-based variance estimation approach to identify genes suited for normalization, applied to bladder and colon cancer data sets. Cancer Res 2004;64:5245-5250.

24 Pfaffl MW: A new mathematical model for relative quantification in real-time RT-PCR. Nucleic Acids Res 2001;29:e45.

25 Chhajlani V: Characterization of a putative alpha-MSH antagonist $153 \mathrm{~N}-6$ at melanocortin receptor subtypes by radioligand binding. Peptides 1996;17:349-351.

26 Schioth HB, Muceniece R, Wikberg JE: Characterization of the binding of MSH-B, HB-228, GHRP-6 and $153 \mathrm{~N}-6$ to the human melanocortin receptor subtypes. Neuropeptides 1997;31:565-571.

27 Menon MB, Ronkina N, Schwermann J, Kotlyarov A, Gaestel M: Fluorescence-based quantitative scratch wound healing assay demonstrating the role of MAPKAPK-2/3 in fibroblast migration. Cell Motil Cytoskeleton 2009;66:1041-1047.

- 28 Lytton J, Westlin M, Hanley MR: Thapsigargin inhibits the sarcoplasmic or endoplasmic reticulum CaATPase family of calcium pumps. J Biol Chem 1991;266:17067-17071.

29 Du P, Kibbe WA, Lin SM: lumi: a pipeline for processing Illumina microarray. Bioinformatics 2008;24:15471548.

30 Ernst J, Bar-Joseph Z: STEM: a tool for the analysis of short time series gene expression data. BMC Bioinformatics 2006;7:191. 


\section{Cellular Physiology Cell Physiol Biochem 2019;52:1339-1360 \begin{tabular}{ll|l} 
and Biochemistry $10.33594 / 000000094$ & $\begin{array}{l}\text { C) } 2019 \text { The Author(s). Published by } \\
\text { Published online: } 4 \text { May } 2019\end{array}$ & Cell Physiol Biochem Press GmbH\&Co. KG
\end{tabular}}

31 Huang DW, Sherman BT, Lempicki RA: Systematic and integrative analysis of large gene lists using DAVID bioinformatics resources. Nat Protoc 2009;4:44-57.

- 32 Supek F, Bosnjak M, Skunca N, Smuc T: REVIGO summarizes and visualizes long lists of gene ontology terms. PLoS One 2011;6:e21800.

- 33 Shannon P, Markiel A, Ozier O, Baliga NS, Wang JT, Ramage D, Amin N, Schwikowski B, Ideker T: Cytoscape: a software environment for integrated models of biomolecular interaction networks. Genome Res 2003;13:2498-2504.

- 34 Merico D, Isserlin R, Stueker O, Emili A, Bader GD: Enrichment map: a network-based method for gene-set enrichment visualization and interpretation. PLoS One 2010;5:e13984.

35 Seidah NG, Benjannet S, Hamelin J, Mamarbachi AM, Basak A, Marcinkiewicz J, Mbikay M, Chretien M, Marcinkiewicz M: The subtilisin/kexin family of precursor convertases. Emphasis on PC1, PC2/7B2, POMC and the novel enzyme SKI-1. Ann N Y Acad Sci 1999;885:57-74.

36 Herraiz C, Garcia-Borron JC, Jimenez-Cervantes C, Olivares C: MC1R signaling. Intracellular partners and pathophysiological implications. Biochim Biophys Acta Mol Basis Dis 2017;1863:2448-2461.

37 Doyle JR, Fortin JP, Beinborn M, Kopin AS: Selected melanocortin 1 receptor single-nucleotide polymorphisms differentially alter multiple signaling pathways. J Pharmacol Exp Ther 2012;342:318-326.

38 Box NF, Wyeth JR, O'Gorman LE, Martin NG, Sturm RA: Characterization of melanocyte stimulating hormone receptor variant alleles in twins with red hair. Hum Mol Genet 1997;6:1891-1897.

- 39 Schioth HB, Phillips SR, Rudzish R, Birch-Machin MA, Wikberg JE, Rees JL: Loss of function mutations of the human melanocortin 1 receptor are common and are associated with red hair. Biochem Biophys Res Commun 1999;260:488-491.

- 40 Herraiz C, Journe F, Ghanem G, Jimenez-Cervantes C, Garcia-Borron JC: Functional status and relationships of melanocortin 1 receptor signaling to the cAMP and extracellular signal-regulated protein kinases 1 and 2 pathways in human melanoma cells. Int J Biochem Cell Biol 2012;44:2244-2252.

41 Adachi S, Nakano T, Vliagoftis H, Metcalfe DD: Receptor-mediated modulation of murine mast cell function by alpha-melanocyte stimulating hormone. J Immunol 1999;163:3363-3368.

42 Barcellini W, Colombo G, La Maestra L, Clerici G, Garofalo L, Brini AT, Lipton JM, Catania A: Alphamelanocyte-stimulating hormone peptides inhibit HIV-1 expression in chronically infected promonocytic U1 cells and in acutely infected monocytes. J Leukoc Biol 2000;68:693-699.

43 Garcia-Borron JC, Sanchez-Laorden BL, Jimenez-Cervantes C: Melanocortin-1 receptor structure and functional regulation. Pigment Cell Res 2005;18:393-410.

- 44 Tsien RY: A non-disruptive technique for loading calcium buffers and indicators into cells. Nature 1981;290:527-528.

- 45 Whang SW, Lee SE, Kim JM, Kim HJ, Jeong SK, Zouboulis CC, Seo JT, Lee SH: Effects of alpha-melanocytestimulating hormone on calcium concentration in SZ95 sebocytes. Exp Dermatol 2011;20:19-23.

- 46 Maresca V, Flori E, Camera E, Bellei B, Aspite N, Ludovici M, Catricala C, Cardinali G, Picardo M: Linking alphaMSH with PPARgamma in B16-F10 melanoma. Pigment Cell Melanoma Res 2013;26:113-127.

- 47 Ha JM, Baek SH, Kim YH, Jin SY, Lee HS, Kim SJ, Shin HK, Lee DH, Song SH, Kim CD, Bae SS: Regulation of retinal angiogenesis by phospholipase C-beta3 signaling pathway. Exp Mol Med 2016;48:e240.

- 48 Eves P, Haycock J, Layton C, Wagner M, Kemp H, Szabo M, Morandini R, Ghanem G, Garcia-Borron JC, Jimenez-Cervantes C, Mac Neil S: Anti-inflammatory and anti-invasive effects of alpha-melanocytestimulating hormone in human melanoma cells. Br J Cancer 2003;89:2004-2015.

49 Suzuki I, Cone RD, Im S, Nordlund J, Abdel-Malek ZA: Binding of melanotropic hormones to the melanocortin receptor MC1R on human melanocytes stimulates proliferation and melanogenesis. Endocrinology 1996;137:1627-1633.

50 Catania A, Colombo G, Carlin A, Garofalo L, Gatti S, Buffa R, Carboni N, Rosso L, Santambrogio L, Cantalamessa L, Lipton JM: Autocrine inhibitory influences of alpha-melanocyte-stimulating hormone in malignant pleural mesothelioma. J Leukoc Biol 2004;75:253-259.

- 51 Weng WT, Huang SC, Ma YL, Chan HH, Lin SW, Wu JC, Wu CY, Wen ZH, Wang EM, Wu CL, Tai MH: alphaMelanocyte-stimulating hormone inhibits angiogenesis through attenuation of VEGF/VEGFR2 signaling pathway. Biochim Biophys Acta 2014;1840:1850-1860.

- 52 Tran PO, Hinman LE, Unger GM, Sammak PJ: A wound-induced [Ca2+]i increase and its transcriptional activation of immediate early genes is important in the regulation of motility. Exp Cell Res 1999;246:319326. 


\section{Cellular Physiology Cell Physiol Biochem 2019;52:1339-1360

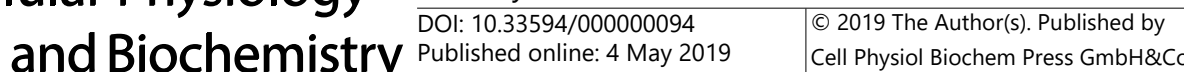 \\ Saporiti et al.: MC1R Enhances Endothelial Cell Migration}

53 Ridley AJ, Schwartz MA, Burridge K, Firtel RA, Ginsberg MH, Borisy G, Parsons JT, Horwitz AR: Cell migration: integrating signals from front to back. Science 2003;302:1704-1709.

- 54 Wei C, Wang X, Zheng M, Cheng H: Calcium gradients underlying cell migration. Curr Opin Cell Biol 2012;24:254-261.

- 55 Mountjoy KG, Kong PL, Taylor JA, Willard DH, Wilkison WO: Melanocortin receptor-mediated mobilization of intracellular free calcium in HEK293 cells. Physiol Genomics 2001;5:11-19.

56 Elliott RJ, Szabo M, Wagner MJ, Kemp EH, MacNeil S, Haycock JW: alpha-Melanocyte-stimulating hormone, MSH 11-13 KPV and adrenocorticotropic hormone signalling in human keratinocyte cells. J Invest Dermatol 2004;122:1010-1019.

57 Bohm M, Apel M, Sugawara K, Brehler R, Jurk K, Luger TA, Haas H, Paus R, Eiz-Vesper B, Walls AF, Ponimaskin E, Gehring M, Kapp A, Raap U: Modulation of basophil activity: a novel function of the neuropeptide alpha-melanocyte-stimulating hormone. J Allergy Clin Immunol 2012;129:1085-1093.

58 Perez DM, Karnik SS: Multiple signaling states of G-protein-coupled receptors. Pharmacol Rev 2005;57:147-161.

59 Yang Y: Structure, function and regulation of the melanocortin receptors. Eur J Pharmacol 2011;660:125130.

60 Breit A, Buch TR, Boekhoff I, Solinski HJ, Damm E, Gudermann T: Alternative G protein coupling and biased agonism: new insights into melanocortin-4 receptor signalling. Mol Cell Endocrinol 2011;331:232-240.

- 61 Munaron L: Intracellular calcium, endothelial cells and angiogenesis. Recent Pat Anticancer Drug Discov 2006;1:105-119.

62 Antigny F, Jousset H, Konig S, Frieden M: Thapsigargin activates Ca(2)+ entry both by store-dependent, STIM1/Orai1-mediated, and store-independent, TRPC3/PLC/PKC-mediated pathways in human endothelial cells. Cell Calcium 2011;49:115-127.

- 63 Clapham DE: Calcium signaling. Cell 2007;131:1047-1058.

- 64 Davis GE, Senger DR: Endothelial extracellular matrix: biosynthesis, remodeling, and functions during vascular morphogenesis and neovessel stabilization. Circ Res 2005;97:1093-1107.

65 Lamalice L, Le Boeuf F, Huot J: Endothelial cell migration during angiogenesis. Circ Res 2007;100:782-794.

- 66 Herbst TJ, McCarthy JB, Tsilibary EC, Furcht LT: Differential effects of laminin, intact type IV collagen, and specific domains of type IV collagen on endothelial cell adhesion and migration. J Cell Biol 1988;106:13651373.

67 Riikonen T, Westermarck J, Koivisto L, Broberg A, Kahari VM, Heino J: Integrin alpha 2 beta 1 is a positive regulator of collagenase (MMP-1) and collagen alpha 1(I) gene expression. J Biol Chem 1995;270:1354813552.

68 Whelan MC, Senger DR: Collagen I initiates endothelial cell morphogenesis by inducing actin polymerization through suppression of cyclic AMP and protein kinase A. J Biol Chem 2003;278:327-334.

69 Skalski M, Yi Q Kean MJ, Myers DW, Williams KC, Burtnik A, Coppolino MG: Lamellipodium extension and membrane ruffling require different SNARE-mediated trafficking pathways. BMC Cell Biol 2010;11:62.

70 Lizama CO, Zovein AC: Polarizing pathways: balancing endothelial polarity, permeability, and lumen formation. Exp Cell Res 2013;319:1247-1254.

71 Schuermann A, Helker CS, Herzog W: Metallothionein 2 regulates endothelial cell migration through transcriptional regulation of vegfc expression. Angiogenesis 2015;18:463-475.

72 Zbinden S, Wang J, Adenika R, Schmidt M, Tilan JU, Najafi AH, Peng X, Lassance-Soares RM, Iantorno M, Morsli H, Gercenshtein L, Jang GJ, Epstein SE, Burnett MS: Metallothionein enhances angiogenesis and arteriogenesis by modulating smooth muscle cell and macrophage function. Arterioscler Thromb Vasc Biol 2010;30:477-482.

73 White SJ, Hayes EM, Lehoux S, Jeremy JY, Horrevoets AJ, Newby AC: Characterization of the differential response of endothelial cells exposed to normal and elevated laminar shear stress. J Cell Physiol 2011;226:2841-2848.

- 74 Yosef N, Regev A: Impulse control: temporal dynamics in gene transcription. Cell 2011;144:886-896.

75 Wu KK, Thiagarajan P: Role of endothelium in thrombosis and hemostasis. Annu Rev Med 1996;47:315331.

76 Tesfamariam B: Endothelial Repair and Regeneration Following Intimal Injury. J Cardiovasc Transl Res 2016;9:91-101. 